archives-ouvertes

\title{
M. Aurelius Cotta Maximus Messallinus : un noble sous les Julio-Claudiens
}

\author{
Cyrielle Landrea
}

\section{To cite this version:}

Cyrielle Landrea. M. Aurelius Cotta Maximus Messallinus : un noble sous les Julio-Claudiens. Mélanges de l'Ecole française de Rome - Antiquité, École française de Rome, 2011, 123 (2), pp.557-579. hal-01830448

\section{HAL Id: hal-01830448 \\ https://hal.archives-ouvertes.fr/hal-01830448}

Submitted on 5 Jul 2018

HAL is a multi-disciplinary open access archive for the deposit and dissemination of scientific research documents, whether they are published or not. The documents may come from teaching and research institutions in France or abroad, or from public or private research centers.
L'archive ouverte pluridisciplinaire HAL, est destinée au dépôt et à la diffusion de documents scientifiques de niveau recherche, publiés ou non, émanant des établissements d'enseignement et de recherche français ou étrangers, des laboratoires publics ou privés. 


\title{
M. Aurelius Cotta Maximus Messallinus : un noble sous les Julio-Claudiens ${ }^{1}$
}

\author{
Cyrielle LANDREA
}

Sous la République, la compétition aristocratique pour le pouvoir était codifiée. Cependant, le $\mathrm{I}^{\mathrm{er}}$ S. av. J.-C. a profondément modifié les mécanismes traditionnels et a ébranlé les certitudes de la nobilitas. À l'issue des guerres civiles, la victoire d'Octavien a marqué la fin des luttes politiques pour le monopole du pouvoir et, par conséquent, a installé l'exercice du pouvoir personnel. Même si l'aristocratie républicaine a été déchirée par les luttes intestines, elle n'en demeure pas moins, à l'avènement du Principat, une composante à part entière du paysage politique, de par son rôle au sein du Sénat. En outre, la restauration octavienne de la res publica passe par le rétablissement d'anciennes valeurs, dont les patriciens sont les porteurs par excellence. Ils sont l'incarnation d'une certaine continuité.

À travers le cas de M. Aurelius Cotta Maximus Messallinus, membre de la gens patricienne des Valerii $^{2}$ et second fils de M. Valerius Messalla Corvinus (cos. 31 av. J.-C.), j'étudierai les marqueurs identitaires de cette noblesse ${ }^{3}$ et son adaptation à la nouvelle donne politique. Selon la tradition, les Valerii sont les descendants de P. Valerius Publicola, un des fondateurs de la République et consul en 509 av. J.-C. ${ }^{4}$ Au ${ }^{\text {er }}$ s., les Valerii Messallae renaissent grâce à la faveur de Sylla ${ }^{5}$ et deux

1. Cet article a bénéficié des relectures critiques de M. DondinPayre, J.-P. Guilhembet et R. Baudry. Je leur exprime toute ma gratitude pour leurs conseils et leurs suggestions. Je reste seule responsable des imperfections qui subsistent.

2. Sur les Valerii, cf. F. Münzer, 1891.

3. Plus globalement sur l'aristocratie augustéenne, voir en dernier lieu Fr. Hurlet, 2009, p. 73-99.

4. $M R R, \mathrm{I}, \mathrm{p} .2$

5. Sylla a épousé une Valeria : Plutarque, Sylla, XXXV, 5-11. Les Valerii Messallae n'ont pas eu de consul de 161 à 61 av. J.-C. La dictature syllanienne a aussi favorisé indirectement les
Valerii Messallae obtiennent le consulat avant l'assassinat de César ${ }^{6}$. Dans le contexte troublé de la République agonisante, M. Valerius Messalla Corvinus, le père de Cotta, fut tour à tour : républicain, partisan d'Antoine ${ }^{7}$ puis partisan d'Octavien. Ainsi, les Valerii participent pleinement au processus de transition conduisant de la République au Principat.

Grâce à son ascendance prestigieuse et au destin politique particulier de son père, le fils de Corvinus, Cotta, né vers 14-13 av. J.-C.8, est un cas intéressant pour appréhender les mutations qui se sont opérées au sein de la nobilitas romaine, à un moment où les élites traditionnelles sont bousculées par l'émergence des élites italiennes, qui s'imposent progressivement depuis l'ère triumvirale. Ces changements sont accentués par les bouleversements politiques liés à l'instauration du Principat. Toutefois, cela n'empêche pas la reconstitution d'une classe politique où les héritiers des antiques familles ont su préserver leur place face à la concurrence des hommes nouveaux. La question de l'adaptation des anciennes familles républicaines, les ruptures et les continuités sont au cour de nos préoccupations. Cotta est un membre de la nobilitas. Son identité est marquée par la uirtus ${ }^{9}$, sa valeur intrinsèque, mais elle doit absolument être

patriciens dans leur ensemble, en augmentant le nombre des titulaires de prêtrises et en affaiblissant les tribuns de la plèbe.

6. M. Valerius Messalla Niger est consul en 61, tandis que son cousin M. Valerius Messalla Rufus l'est en 53.

7. M.-C. Ferriès, 2007, n 138.

8. Cette datation est proposée en admettant l'obtention de son consulat suo anno. U. Vogel-Weidemann (1982, p. 283, note 525) fait le point sur les différentes hypothèses.

9. Ovide, Pontiques, II, 3, 1-6 : Maxime, qui claris nomen uirtutibus aequas/ Nec sinis ingenium nobilitate premi. 
mise en relation avec ses ascendants. Cornutus rappelle ainsi que parmi ses ancêtres, un avait géré sept fois le consulat ${ }^{10}$. La mémoire collective romaine faisait du critère de l'exercice des magistratures supérieures le fondement même de la noblesse ${ }^{11}$. De surcroît, Cotta appartient au patriciat, la «nobilitas par excellence $»^{12}$. Sa naissance dans la gens des Valerii lui a conféré une légitimité spécifique. Il s'agit donc d'identifier les permanences et les ruptures dans la définition de l'identité nobiliaire, à travers l'exemple précis de Cotta, né sous le Principat d'Auguste.

Après avoir envisagé les problèmes d'identification spécifique au personnage, l'enquête porte sur plusieurs marqueurs de l'identité nobiliaire ${ }^{13}$ et aristocratique, à commencer par l'importance de la famille et la généalogie prestigieuse. Les nobles se définissent aussi en fonction de critères politicoreligieux et Cotta ne déroge pas à la règle. Ainsi, sa carrière, son rôle politique au Sénat et ses sacerdoces sont abordés, sans oublier la mutation d'une partie de la noblesse républicaine en noblesse impériale, dont l'incarnation la plus visible est l'émergence des amici principis. Cotta Messallinus n'a jamais caché son amitié avec Tibère, une amitié assez puissante pour le protéger d'éventuelles poursuites judiciaires. En outre, il ne faut pas omettre les fondements économiques et sociaux de l'identité nobiliaire. Le patrimoine, la clientèle et le patronat sont autant de marqueurs définissant la noblesse. Enfin, la dimension litté-

10. Commentum Cornuti in Persium in Satiram II, 71-72. Voir note 105.

11. Chr. Badel, 2005, p. 16.

12. R. Baudry, 2006, p. 171

13. L'appartenance de Cotta à la nobilitas était une évidence aux yeux des contemporains (Ovide, Pontiques, III, 2, 103-110) : Adde quod est animus semper tibi mitis et altae/ Indicium more nobilitatis habent. Ce cas est d'autant plus intéressant que le poète met en avant la "double noblesse" de Cotta : Te tamen in turba non ausim, Cotta, silere,/ Pieridum lumen praesidiumque fori, / Maternos Cottas cui Messalasque paternos, / Maxime, nobilitas ingeminata dedit (Ovide, Pontiques, IV, 16, 41-44). Il est issu d'une gens patricienne séculaire du côté paternel, mais a des ascendants maternels faisant partie de la noblesse plébéienne. Sur les identités patricienne et nobiliaire, voir R. Baudry, 2006, p. 155-167.

14. Annales et chronica aeui Salici, Mariani Scotti chronicon, p. 486 et 501 .

15. Chronique mineure, Cassiodori senatoris chronica, p. 136; CIL, VI, 10051 et Tacite, Annales, III, 2, 3.

16. Tacite, Annales, III, 17, 4.

17. CIL, VI, 32270 et XIV, 244. Sur un fragment des fastes des saliens palatins : M. Aure[lius Cotta] (C. Letta, 2000, p. 521539). raire et culturelle est particulièrement présente chez les Valerii Messallae, participant entre autres à la postérité de Cotta Messallinus.

\section{LES DÉNOMINATIONS D'UN NOBLE}

Ses dénominations antiques sont pléthoriques. Avant son adoption, il s'appelait Valerius Messalla, sans que le praenomen soit connu. Après son adoption par un membre de la gens des Aurelii, l'onomastique du personnage est plus complexe. Même si l'onomastique est codifiée, elle jouit néanmoins d'une certaine souplesse et la diversité rencontrée dans les témoignages épigraphiques et littéraires l'illustre parfaitement.

La forme la plus succincte est celle du nomen Aurelius ${ }^{14}$, avec addition éventuelle du praenomen Marcus ${ }^{15}$. Toutefois, les mentions les plus fréquentes indiquent le cognomen Cotta: Aurelius Cotta $^{16}$ ou M. Aurelius Cotta ${ }^{17}$, mais un autre cognomen est possible, M. Aurelius Maximus ${ }^{18}$. Plusieurs cognomina peuvent apparaître : $M$. Aurelius Cotta Maximus ${ }^{19}$, voire M. Aurelius Cotta Maximus Messallinus ${ }^{20}$. Certaines sources le signalent simplement par un cognomen : $\operatorname{Cotta}^{21}$ ou Maximus $^{22}$. Enfin, la juxtaposition de deux cognomina est attestée : Messallinus Cotta ${ }^{23}$, Cotta Messallinus $^{24}$, Cotta Maximus ${ }^{25}$ et Maximus Cotta ${ }^{26}$. Notons que les sources tardives avancent une orthographe quelque peu approximative : Gotta ${ }^{27}$ et Grato $^{28}$.

18. Commentum Cornuti in Persium in Satiram, II, 71-72.

19. AE, 1983, 515 (Tabula Siarensis); AE, 1985, 52 et CIL, XIV, 2298.

20. Forschungen in Ephesos, III, p. 112, 22 et Sardis, VII, 1, 36.

21. Chronique mineure, Fasti consulares a regibus exactis ad a. 354 , p. 280 et Chronique mineure, V. Consules, p. 502; Digeste, I, 16, 4, 2 et XLVIII, 2, 12, 3; Fasti Vindobonenses priores; Juvénal, Satires, V, 109 et VII, 95; Ovide, Pontiques, III, 2 (destinataire), 1 et IV, 16, 41; Tacite, Annales, VI, 5, 2 et VI, 7, 1 et Velleius Paterculus, Histoire romaine, II, 112, 1-2.

22. Ovide, Pontiques, I, 5, 2 et 9; I, 9, 16, 26, 27, 32 et 50; II, 3, 1; III, 5, 34, 58 et IV, 16, 44.

23. Commentum Cornuti in Persium in Satiram II, 71-72; Pline l'Ancien, Histoire naturelle, X, 27, 52 et Tacite, Annales, IV, 20, 4.

24. Pline l'Ancien, Histoire naturelle, praefatio des livres XIV et XV; Tacite, Annales, II, 32, 1; V, 3, 2; VI, 5, 1 et XII, 22, 2.

25. Cotta Maximus est désigné comme destinataire de plusieurs lettres d'Ovide : Pontiques, I, 5; I, 9; II, 3; II, 8 et III, 5.

26. Ovide, Pontiques, II, 8, 2 et III, 5, 6.

27. Chronique mineure, Fasti consulares a regibus exactis ad a. 354, p. 280, plus particulièrement Fasti Vindobonenses posteriores.

28. Hydatii episcopi descriptio consulum ex quo primum ordinati sunt, 20 . 
Ainsi, il se nomme M. Aurelius Cotta Maximus $^{29}$, puis M. Aurelius Cotta Maximus Messallinus après la mort de son frère ${ }^{30}$, survenue peu après 21 ap. J.-C. La formule officielle des tria nomina peut être allongée ou allégée. La manière dont Ovide le désigne montre incontestablement que les usages quotidiens et l'amitié adaptent la formule officielle, conférant une plus grande proximité entre les deux personnes. Enfin, ce sénateur porte l'héritage des Aurelii Cottae et des Valerii Messallae à travers son onomastique.

Ces différentes dénominations ont troublé les historiens. Dans son récit a posteriori, Tacite anticipe sur l'onomastique plus tardive, en appelant l'aristocrate Cotta Messallinus, alors qu'il ne portait pas ce deuxième cognomen à l'époque de l'événement mentionné. Certains ont cru pouvoir distinguer deux personnages, partant du principe qu'il y avait une trop grande différence d'âge entre Messallinus (cos. 3 av. J.-C.) et le consul de 20. Néanmoins, Klebs ${ }^{31}$, dans la première version de la Prosopographia imperii romani, avait déjà identifié Cotta Messallinus et Aurelius Cotta (cos. 20 ap. J.-C.), s'appuyant sur des passages des lettres d'exil d'Ovide où Cotta est qualifié de iuuenis ${ }^{32}$. Dans la deuxième version de la PIR, Groag a bien pris acte des arguments de Klebs, mais distingue toutefois deux personnages. M. Aurelius Cotta ${ }^{33}$ serait le fils adoptif de Cotta Messallinus ${ }^{34}$. Il fallut attendre un article de 1937 signé par De Laet ${ }^{35}$, puis un autre par Degrassi ${ }^{36}$ pour affirmer à nouveau l'existence d'un seul homme, unicité qui fait l'objet d'un consensus depuis la découverte de la Tabula Siarensis, où le consul de 20 est nommé M. Aurelius Cotta Maximus ${ }^{37}$.

29. Ce cognomen, utilisé par la gens des Valerii, rappelle son passé prestigieux. Un des frères de Valerius Publicola s'appelait $M^{\prime}$. Valerius Maximus : H. Volkmann, $R E$, Valerius $\mathrm{n}^{\circ} 243$, 1955, col. 116-120. Puis ce cognomen fut notamment porté par $\mathrm{M}^{\prime}$. Valerius Maximus (cos. 263 av. J.-C.), le vainqueur de Messine (Macrobe, Saturnales, I, 26) : F. Münzer, Valerius $\mathrm{n}^{\circ} 247$, col. 123-125.

30. Velleius Paterculus, Histoire romaine, II, 112, 2 : cognomen suum Cottae fratri relinqueret.

31. E. Klebs, PIR $^{1}, 1897, \mathrm{~A}, \mathrm{n}^{\circ} 1236$.

32. Ovide, Pontiques, II, 3, 55 et III, 5, 7.

33. E. Groag, PIR $2,1933, \mathrm{n}^{\circ} 1487$.

34. Ibid., $\mathrm{n}^{\circ} 1488$.

35. S. J. De Laet, 1937, p. 138-140.

36. A. Degrassi, 1946, p. 38.

37. Tabula Siarensis, fragment b, colonne II, 1. 27-28. Cette identification indubitable a été montrée par O. Salomies
L'APPARTENANCE À LA GENS DES VALERII ${ }^{38}$

\section{Les ancêtres}

D'origine sabine, cette famille patricienne fut le témoin actif de la chute de la royauté et de l'instauration de la République. P. Valerius Publicola ${ }^{39}$, $l^{\prime}$ «ami du peuple», en est un des fondateurs ${ }^{40}$. Une autre figure, quasi mythique, rehausse encore le prestige de la gens, M. Valerius Corvus ${ }^{41}$. La tradition raconte qu'au milieu du IV ${ }^{\text {e }}$ s., celui-ci défie un guerrier gaulois en combat singulier et un corbeau se pose sur le casque de Valerius, l'aidant à terrasser le Gaulois ${ }^{42}$. Cet acte de bravoure a fait la renommée de ce personnage, lui permettant de gérer le consulat à vingt-trois ans ${ }^{43}$, et a entraîné le port du cognomen de Coruus ou de Coruinus par la famille. Quelques décennies plus tard, $\mathrm{M}^{\prime}$. Valerius Maximus $^{44}$ (cos. 263 av. J.-C.) a vaincu la cité de Messine lors de la première guerre punique. Sa gloire fut telle qu'il adjoignit à son nom le cognomen de Messalla ${ }^{45}$. Ces deux cognomina sont autant héroïques qu'ancrés dans l'histoire de Rome. Pour rappeler son ascendance illustre, le jeune aristocrate a d'ailleurs pris le cognomen de Maximus, avant d'adjoindre, plus tard, celui de Messallinus. La compétition mémorielle est importante dans la définition des élites et les Romains pouvaient se fabriquer ou rappeler une généalogie prestigieuse : les élites étaient les dépositaires de la mémoire collective et formaient les figures historiques. Dans un tel contexte, les prétentions généalogiques des Valerii Messallae ont été érigées en savoir antiquaire, particulièrement par Valerius Antias, mais aussi par le père de Cotta, Messalla

(1992, p. 14).

38. Un tableau généalogique simplifié de la famille des Valerii Messallae se trouve en annexe.

39. H. Volkmann, RE, Valerius no 302, col. 180-189.

40. Une inscription archaïque a été découverte sur l'ancien site de Satricum en 1977. Elle mentionne une dédicace au dieu Mars réalisée par les suodales d'un certain Poplios Valesios. Sans prouver l'existence de Valerius Publicola, elle permet de montrer l'importance des Valerii au début de la République, se reporter à C. M. Stibbe, G. Colonna, C. De Simone et H. C. Versnel, 1980.

41. H. Volkmann, RE, Valerius $n^{\circ} 137$, col. 2413-2418.

42. MRR, I, p. 129.

43. Tite-Live, Histoire romaine, VII, 26, 12.

44. F. Münzer, RE, Valerius $n^{\circ} 247$, col. 123-125.

45. Macrobe, Saturnales, I, 6, 26; Sénèque, De la brièveté de la vie, XIII, 5. 
Corvinus, indigné par les fausses généalogies et désireux de distinguer ses origines illustres de celles des imposteurs ${ }^{46}$. Par ailleurs, Auguste fit élever une statue de Valerius Corvus sur son forum $^{47}$, afin d'exalter le prestige de la gens Valeria.

Ovide ne manque pas de louer l'ascendance illustre de Cotta, créant un lien entre la nobilitas et la uirtus. En rendant ainsi hommage à la grandeur de la gens des Valeriii ${ }^{48}$, le poète souhaite amadouer les enfants de Messalla, afin qu'ils agissent auprès de l'empereur. Ces revendications généalogiques s'ancrent dans la mémoire civique romaine et renforcent les prétentions patriciennes. Même si ces prétentions ne sont pas l'apanage des patriciens, mais plutôt une caractéristique de l'identité nobiliaire, les patriciens peuvent se vanter d'une plus grande ancienneté et faire remonter leurs origines jusqu'à les faire coïncider avec la naissance de l'Vrbs.

\section{La parenté}

Son père est $M$. Valerius Messalla Corvinus ${ }^{49}$. Ce dernier est le fils de Messalla Niger (cos. 61 av. J.-C.), défenseur de la cause républicaine et membre de la faction des optimates. Corvinus est un ami de Brutus et un disciple de Cicéron. À la mort de César, le patricien est âgé d'à peine vingt ans. Ses convictions sont clairement républicaines et il est inscrit sur la liste des proscriptions de 43. Ensuite, il devient un partisan d'Antoine pour finalement rejoindre le camp octavien, gérant le consulat en 31, à la place d'Antoine. Enfin, son attitude demeure ambiguë durant le Principat. Il démissionna de la préfecture urbaine en $26^{50}$, mais honora Auguste, au nom du

46. Pline l'Ancien, Histoire naturelle, XXXV, 8.

47. Aulu-Gelle, Nuits attiques, IX, 11, 10.

48. Ovide, Pontiques, III, 2, 105. Indubitablement cette référence à Volesus, fondateur mythique de la gens, atteste la fascination d'Ovide pour cette famille. Le poète a été remarqué à ses débuts par Messalla Corvinus (Ovide, Pontiques, II, 3 , 75-78). Un autre passage est à mentionner: Ovide, Pontiques, III, 2, 103-108.

49. Messalla Corvinus est de loin le personnage le plus important de la famille à la fin de la République et au début du Principat. La bibliographie le concernant est donc considérable. Nous renvoyons à quelques notices : R. Hanslik, RE, Valerius $n^{\circ}$ 261, 1955, col. 131-157; J. Scheid, 1975, p. 50-57 et M.-C. Ferriès, 2007, n ${ }^{\circ} 138$. Concernant les attitudes à l'égard d'Auguste et les changements d'allégeance, voir notamment : J. L. Butrica, 1994, p. 279-296 et P. Tansey, 2007, p. 882-890.

50. Jérôme, Chronique, 164, f (Helm) : Messala Coruinus primus
Sénat, du titre de pater patriae en 2 av. J.-C. ${ }^{51}$ Fin lettré, grand orateur, écrivain à ses heures, il est également connu pour avoir animé un cercle littéraire de la même envergure que celui de Mécène ${ }^{52}$.

La mère de Cotta est une Aurelia. Cette dernière semble être encore vivante en 13 ap. J.-C., selon Ovide ${ }^{53}$. Elle est probablement la fille de M. Aurelius Cotta, préteur en 54 et époux d'une Calpurnia. Mais selon R. Syme ${ }^{54}$, la mère de Cotta pourrait tout aussi bien être une Calpurnia, fille d'un Calpurnius marié à une Aurelia. Ainsi, Cotta aurait été adopté non par le frère de sa mère, mais par l'oncle de celle-ci. Le premier fils de Corvinus connu est M. Valerius Messalla Messallinus (cos. 3 av. J.-C. $)^{55}$. Cotta reprit le cognomen de son aîné, lorsque celui-ci mourut. À partir de ce moment, il est plus connu sous le nom de Cotta Messallinus. Il a aussi une sœur, Valeria Messallina ${ }^{56}$.

Valeria Messallina se marie avec T. Statilius Taurus (cos. 11 ap. J.-C.). De leur union sont nés Statilius Taurus (cos. 44 ap. J.-C.) et Taurus Statilius Corvinus $^{57}$ (cos. 45 ap. J.-C.). Le consul de 44 est probablement le père de Statilia Messallina, épouse de Néron.

Les Valerii Messallae s'acquittent naturellement de leurs obligations envers les ancêtres par la perpétuation de leur souvenir. Cet aspect mémoriel a été autrefois associé au prétendu tombeau de Messalla Corvinus, connu sous le nom de Casal Rotondo. C'est le plus grand mausolée cylindrique construit le long de la Via Appia. Au XIX ${ }^{\text {e }}$ s., Luigi Canina fut chargé des fouilles et un fragment d'inscription a été retrouvé, mentionnant seulement Cotta $^{58}$. Des hypothèses ont été formulées,

praefectus Vrbi factus sexto die magistratu se abdicauit inciuilem potestatem esse contestans.

51. Suétone, Auguste, LVIII, 1-2.

52. Sur tous ces aspects, voir notamment A. Valvo, 1983 p. 1663-1681.

53. Ovide, Pontiques, II, 3, 73.

54. Ibid.,, III, 2, 103-110. Sur ce point, consulter, R. Syme, 1978, p. 120-121.

55. Velleius Paterculus, Histoire romaine, II, 112, 2.

56. FOS, $\mathrm{n}^{\circ} 773$. M.-Th. Raepsaet-Charlier propose un second mariage dont serait issue Vedusia $\left(\mathrm{n}^{\circ} 785\right)$. Nous trouvons également la forme raccourcie de Messallina à la place de Valeria Messallina. Nous ignorons si elle est la sœur aînée ou cadette de Cotta. Sa place dans le stemma est donc arbitraire.

57. Suétone, Claude, XIII, 3.

58. CIL, VI, 1395. Le fragment est dorénavant visible sur la façade du mausolée. 


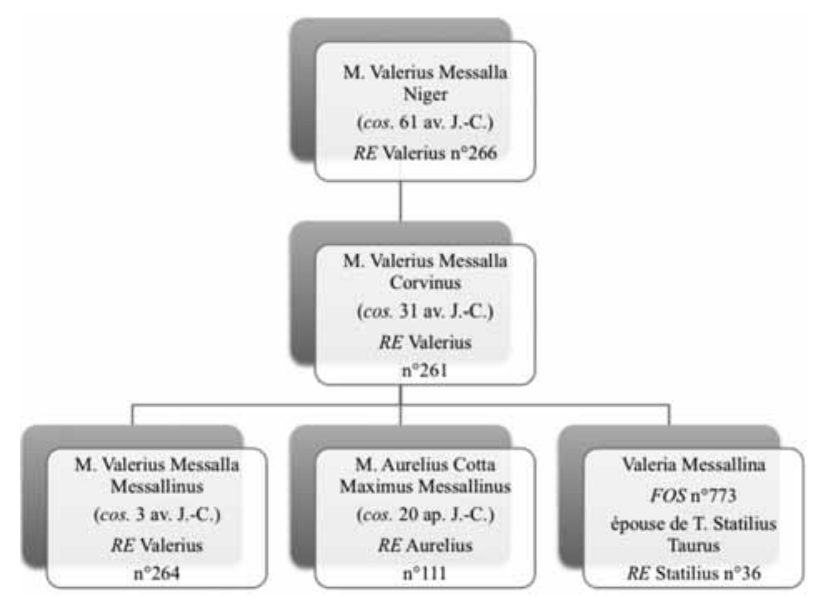

Fig. 1 - Descendance simplifiée de M. Valerius Messalla Niger.

afin d'identifier le commanditaire du tombeau et la personne honorée. Les sources latines mentionnent un tombeau d'un membre de la gens. À deux reprises, Martial cite des saxa ${ }^{59}$ et marmora ${ }^{60}$ Messallae. Le poète parle probablement du tombeau de Messalla Corvinus, le père de Cotta. La première occurrence ${ }^{61}$ doit exprimer la survie audelà de la mort des vers de Martial. Plus de quatrevingts ans après sa mort, sa tombe était encore renommée. La seconde occurrence offre un parallèle entre le tombeau de Messalla et celui de C. Passienus Crispus ${ }^{62}$. Ce sont les outrages du temps qui sont au cœur du passage car le tombeau aurait été attaqué par la croissance d'un figuier. L. Canina a tenté de retracer l'histoire du Casal Rotondo, grâce à l'aide de Borghesi ${ }^{63}$. Selon L. Canina, ce serait le tombeau de Messalla Corvinus, décrit par Martial, et l'inscription désignerait le commanditaire. Une restitution a même été proposée : [M. Aurelius M. f. M. n.] Cotta / [Messalae Coruino patri] ${ }^{64}$. Cette hypothèse séduisante ne peut pourtant pas être retenue ${ }^{65}$, car l'étude des éléments conservés fait plutôt penser au début du

59. Martial, Epigrammes, VIII, 3, 5.

60. Ibid., X, 2, 9.

61. Ibid., VIII, 3, 5 : et cum rupta situ Messallae saxa iacebunt.

62. Ibid., X, 2, 9-10: Marmora Messallae findit caprificus et audax/ Dimidios Crispi mulio ridet equos.

63. L. Canina, 1853, p. 151.

64. Ibid., p. 152. Il donne une première restitution non retenue ici : [Marcus Valerius Messal[l]inus] Cotta [Messal[l]ae Coruino patri VII mille].

65. Voir le résumé par M. Macciocca, 2004, p. 166.

66. Il faut rester prudent car les tombeaux peuvent être restaurés et les inscriptions refaites, rendant ainsi plus difficile

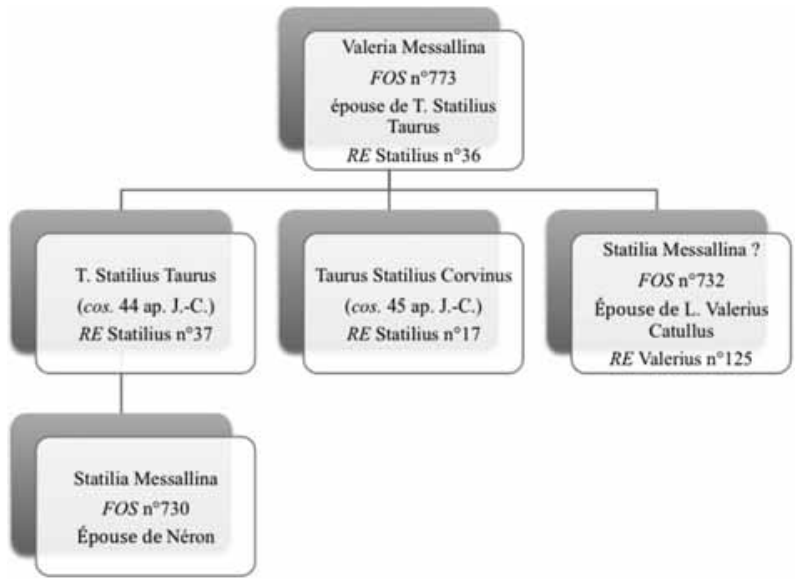

Fig. 2 - Descendance simplifiée de Valeria Messallina.

Principat d'Auguste ${ }^{66}$. Plusieurs identifications sont possibles: L. Aurelius Cotta ${ }^{67}$, M. Aurelius Cotta $^{68}$ ou encore Aurelius Cotta, légat en Sardaigne en 49 av. J.-C. ${ }^{69}$ Toutefois, rien ne justifie pleinement cette attribution et ce fragment demeure un indice bien trop mince pour trancher en faveur d'un mausolée commandité par Cotta.

\section{LA DESCENDANCE}

Un faisceau d'indices permet d'en esquisser les contours. Sa ou ses épouses ne sont pas connues. Ovide mentionne l'existence d'une d'entre elles, mais sans la nommer ${ }^{70}$. En 11, le noble est donc marié. Étant né vers 14 av. J.-C., il se serait marié environ à l'âge de 20 ans. Dans les Tristes, le poète loue avec emphase la progéniture de $\operatorname{Cotta}^{71}$, qui aurait eu au moins une fille et un garçon avant 11. Essayons maintenant de les identifier.

La descendance féminine est hypothétique, mais une vestale dénommée Aurelia Cotta ${ }^{72}$ figure sur une inscription $\mathrm{d}^{\prime}$ Athènes ${ }^{73} \mathrm{du}$ deuxième quart $\mathrm{du} \mathrm{I}^{\mathrm{er}}$ s. ap. J.-C. ${ }^{74}$ Cette inscription n'im-

la tentative de datation.

67. P. von Rohden, $R E$, Aurelius $\mathrm{n}^{\circ} 102$, col. 2485-2487.

68. Ibid., Aurelius n ${ }^{\circ}$ 108, col. 2489.

69. E. Groag, PIR $2,1933, \mathrm{n}^{\circ} 1485$.

70. Ovide, Tristes, IV, 5, 27-28.

71. Ibid., IV, 5, 31-34.

72. FOS, $\mathrm{n}^{\circ} 131$.

73. $I G, \mathrm{II}^{2}, 3534$.

74. M. Kajava, 1990, p. 76. Le fait d'honorer des vestales à Athènes ne serait pas anodin. En effet, c'est lié au culte d'Athéna Parthénos, régulièrement vue comme une vierge. Pour de plus amples informations sur les rapports entre les 
plique pas la venue de la vestale à Athènes, puisque son devoir était de veiller au feu sacré romain et empêchait de tels voyages ${ }^{75}$. Le $\delta \tilde{\eta} \mu \mathrm{s}$ de la cité décide de lui ériger une statue avec une inscription honorifique, afin de louer sa piété en tant que vestale. Il pourrait ainsi s'agir de la fille de Cotta, qui n'a pas forcément de rapport avec Athènes ${ }^{76}$, mais sa position de proconsul d'Asie aurait pu lui permettre de se faire connaître des Athéniens ${ }^{77}$. L'inscription aurait pu être commanditée par le père s'il servait en Grèce, mais ce n'est pas le cas. L'érection d'une telle statue à Athènes pourrait être due au prestige des ancêtres de la vestale $^{78}$. Or, les Valerii Messallae ont des liens étroits avec l'Orient romain. Le grand-père paternel de la vestale y termina ses études ${ }^{79}$, mais $c^{\prime}$ est probablement une coïncidence. Cotta est aussi proconsul d'Asie vers 35/36, l'inscription pourrait ainsi dater de cette époque. Les Athéniens auraient honoré une vestale, fille du proconsul $\mathrm{d}^{\prime}$ Asie alors en charge. Une autre inscription ${ }^{80}$ mentionne une lectrix. Cet esclave Derceto pourrait être la lectrix d'Aurelia, c'est-à-dire de la vestale, qui serait née au début du $\mathrm{I}^{\text {er }} \mathrm{s}$. ap. J.-C. et serait morte vraisemblablement après $30^{81}$.

Aulu-Gelle rappelle les conditions pour devenir vestale et, parmi celles-ci, il faut que le père ait moins de trois enfants ${ }^{82}$ et que les deux parents soient encore vivants ${ }^{83}$. Ainsi, Cotta n'eut probablement que deux enfants viables, la vestale Aurelia et Aurelius Cotta. Ce dernier est peut-être celui mentionné par Tacite ${ }^{84}$. Cet aristocrate a dilapidé la fortune familiale et l'empereur Néron lui a octroyé une rente annuelle, afin de maintenir son patrimoine. Il faut aussi relever que Tacite cite, dans le même passage, un autre descendant de Messalla Corvinus, son homonyme, consul de 58, qui reçut 500000 sesterces par an. Cotta pourrait

vestales et Athènes, se reporter à M. Kajava, 2001, p. 71-94. 75. M. Kajava, 2001, p. 73.

76. Selon P. Graindor (1931, p. 45), une délégation de vestales aurait été reçue à Athènes pour des cérémonies religieuses. Cette proposition ne sera pas retenue car les vestales ne semblent pas avoir quitté Rome.

77. J. H. Oliver, 1971, p. 55 : le proconsul d'Asie était très bien connu des Athéniens, compte tenu des relations qui existent entre la province asiatique et des cités comme Athènes. L'auteur place le proconsulat de Cotta sous Tibère. Nous reviendrons ultérieurement sur la datation de cette promagistrature.

78. M. Kajava, 1990, p. 76

79. Cicéron, Lettre à Atticus, XII, 32, 2. La lettre date du 28 mars d'ailleurs être l'homme de lettres mentionné par Juvénal ${ }^{85}$. En effet ce passage cite un Pison, identifié à C. Calpurnius Piso. Complice d'une conjuration contre Néron, il est obligé de se suicider en 65. De fait, il paraît logique de croire que ce Pison et Cotta vivent à la même époque. La générosité de Cotta a dû marquer les contemporains, car il est plus âgé que les deux autres personnages. Dans ce cas, Cotta aurait laissé le souvenir d'un homme aux largesses mémorables, même plusieurs décennies après sa mort. Mais selon J. Ferguson ${ }^{86}$, il pourrait plutôt s'agir du fils du consul de 20 qui s'appellerait alors Aurelius Cotta, pouvant être identifié à celui mentionné par Martial ${ }^{87}$. Le patronat est un des moyens de dilapider son héritage et son patrimoine. L'allusion à un Cotta mort forcerait à penser qu'il en existe encore un à l'époque de Juvénal et de Martial. Mis à part ces deux descendants directs, on a repéré une descendante plus indirecte, Lollia Paulina, petite-nièce par son père de Cotta Messallinus ${ }^{88}$.

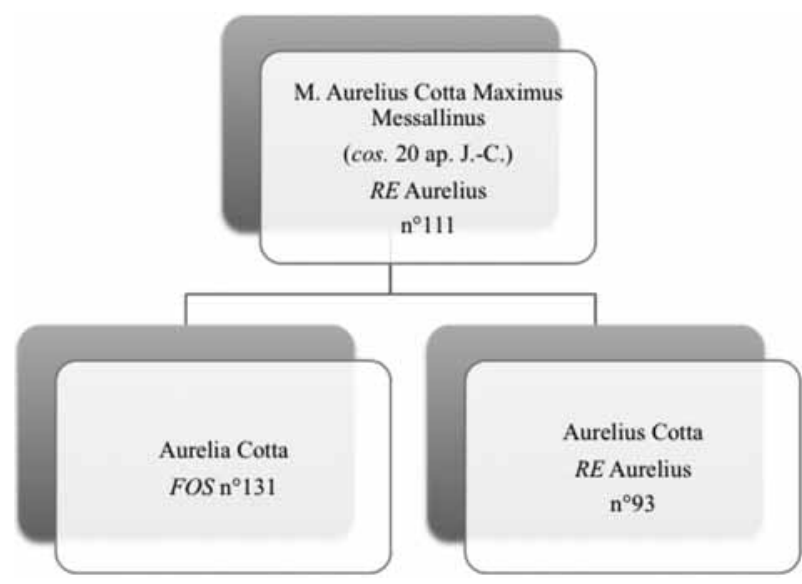

Fig. 3 - Descendance simplifiée de M. Aurelius Cotta Maximus Messallinus.

45 av. J.-C.

80. CIL, VI, 33473.

81. J. Rüpke, 2005, p. 795 (tome 2). J. C. Saquete avait déjà émis cette hypothèse en concluant qu'elle avait dû vivre dans la première moitié du $I^{\text {er }}$ s. ap. J.-C. (J. C. Saquete, 2000, p. 138)

82. Aulu-Gelle, Nuits attiques, I, 12, 8.

83. Ibid., I, 12, 2.

84. Tacite, Annales, XIII, 34.

85. Juvénal, Satires, V, 109-110. C'est l'opinion de E. Groag $\left(P I R^{2}\right.$, 1933, no 1486).

86. J. Ferguson, 1987, p. 69

87. Martial, Epigrammes, VI, 70.

88. Tacite, Annales, XII, 22, 1-2. 
LES STRATÉGIES FAMILIALES :

L'ALLIANCE AVEC LES AURELII COTTAE

L'onomastique de l'aristocrate indique clairement qu'il fut adopté, puisque nous retrouvons le nomen Aurelius et le cognomen Cotta. L'adopté a pris le praenomen Marcus et le nomen Aurelius de l'adoptant. Le praenomen Marcus est très répandu chez les Valerii, mais il était déjà porté par son frère aîné Messallinus. Il aurait pu alors s'appeler Manius ou Lucius, également attestés dans la famille des Valerii Messallae à cette époque. En outre, l'adopté porte généralement un cognomen rappelant sa famille d'origine. C'est pourquoi le noble garde celui de Maximus et ajoute Cotta pour rappeler l'adoption. Le premier cognomen seréfère la grandeur de sa gens d'origine. Son adoption s'inscrit certes dans un contexte de déclin démographique d'une frange de la noblesse, mais qui n'atteint pas les Valerii. La création de nouveaux patriciens par César et Auguste n'avait pas enrayé le déclin démographique des anciennes familles patriciennes. Dans le cas qui nous intéresse, pourtant, l'adoption d'un patricien par une famille plébéienne atteste la vitalité de la gens des Valerii et $c^{\prime}$ est un honneur pour les Aurelii ${ }^{89}$. Sans descendance mâle, les Aurelii Cottae étaient voués à l'extinction ${ }^{90}$.

Néanmoins, un doute plane sur le nouveau statut de Cotta. En effet, gardait-il son statut de patricien ou devenait-il un plébéien? Les arguments traditionnellement avancés paraissent dorénavant obsolètes. C. Letta a récemment identifié Cotta comme un membre de la confrérie des

89. Parmi les patriciens adoptés par les plébéiens, citons l'exemple de Claudius Pulcher, adopté par M. Livius Drusus au I ${ }^{\text {er }}$ S. av. J.-C. (Suétone, Tibère, III, 1-2). Pour de plus amples informations, se reporter à M.-H. Prévost, 1949, p. 18-29. L'exemple le plus connu sous la République est celui de P. Clodius Pulcher, où la perte du statut de patricien était le but recherché. Sous le Principat d'Auguste, un cas d'adoption d'un patricien par une famille plébéienne est attesté. Il s'agit de Q. Caecilius Metellus Creticus Silanus (E. Groag, $R E$, Caecilius $\mathrm{n}^{\circ}$ 90, col. 1212).

90. Ovide, Pontiques, III, 2, 103-110.

91. C. Letta, 2000, p. 521-539. Nous reviendrons ultérieurement sur cette inscription. Voir p 26. Pour justifier le statut de patricien, C. Letta (p. 535) fait appel à un passage de Pline l'Ancien, mentionnant un Messallinus e consularibus patriciis (Pline l'Ancien, Histoire naturelle, XXXII, 123). Or, il ne s'agit absolument pas de Cotta, mais de son frère aîné, le consul de 3 av. J.-C. Dans l'édition C.U.F., E. de Saint-Denis l'identifie à un hypothétique M. Aurelius Cotta Messalinus (cos. 74 av. J.-C.) : E. de Saint-Denis, 1966, p. 124. Nous ne saliens palatins ${ }^{91}$. Or, les douze membres de ce collège sont des patriciens ${ }^{92}$. En outre, au moment de la cooptation, ils doivent être patrimus et matrimus, c'est-à-dire, que les deux parents doivent être en vie, ce qui est le cas, puisque l'inscription des saliens pourrait dater de 4 ap. J.-C. ${ }^{93}$. L'adoption d'un patricien par une famille plébéienne n'impliquait pas forcément la perte du statut à l'époque impériale. Existe-t-il d'autres exemples de patriciens adoptés par des plébéiens, mais dont le statut juridique resta inchangé? Ceci semble être le cas de Ti. Plautius Silvanus Ælianus, faisant probablement partie des Alii Lamiae et adopté par un M. Plautius Silvanus, soit le consul de 2 av. JC. ou plus vraisemblablement son fils, le préteur de 24 ap. J.-C. ${ }^{94}$ Une inscription ${ }^{95}$ livre sa brillante carrière et notamment le début typique de celle $d^{\prime}$ un patricien ${ }^{96}$. La perte du statut de patricien n'est donc pas automatique et Cotta aurait pu demeurer un patricien après son adoption.

Le père adoptif appartient à la famille des Aurelii Cottae, descendante du consul de 252. Plusieurs hypothèses peuvent être avancées à son sujet. Ce père pourrait être $\mathrm{M}$. Aurelius Cotta ${ }^{97}$, légat en Sardaigne en 49 av. J.-C., chassé de l'île par les habitants, avant l'arrivée du légat de César et qui se réfugia ensuite en Afrique ${ }^{98}$. Mais il aurait dû vivre longtemps pour pouvoir adopter le fils de Corvinus ${ }^{99}$. Existe-t-il d'autres possibilités? Valère Maxime fait allusion au fils de $M$. Cotta (cos. 74 av. J.-C.) qui poursuivit l'accusateur de son père, C. Papirius Carbo, le jour même de sa prise de la toge virile, sous le chef de concussion ${ }^{100}$.

pouvons pas suivre E. de Saint-Denis sur ce point, puisque le consul de 74 est M. Aurelius Cotta.

92. Juvénal, VI, 604. Le poète ne le dit pas explicitement, mais le nom des Scauri est utilisé pour désigner l'ancienneté des familles des saliens, donc des gentes patriciennes. Le monopole des patriciens sur ce sacerdoce est également notamment attesté par Cicéron, Sur sa maison, XXXVIII et Lucain, Pharsale, IX, 477-480.

93. C. Letta, 2000, p. 537.

94. Pour de plus amples informations sur le cas de Ti. Plautius Silvanus Aelianus, voir L. Ross Taylor, 1956, p. 28-29.

95. CIL, XIV, 3608.

96. Il est IIIuir A.A.A. F.F. et q. Ti. Caesaris. Ensuite, il n'a été ni tribun, ni édile (M. Hofmann, 1951, col. 35). Il a été questeur du prince, ce qui pourrait être mis en parallèle avec l'hypothèse de ce type de questure exercé par Cotta.

97. P. von Rohden, RE, Aurelius no 109, col. 2489.

98. Dion Cassius, Histoire romaine, XLI, 18, 1.

99. E. Groag, $P I R^{2}, 1933, \mathrm{n}^{\circ} 1485$.

100. Valère Maxime, Faits et dits mémorables, V, 4, 4 
De fait, il est trop jeune pour gouverner la Sardaigne en 49. C'est un autre Aurelius Cotta, donc un autre père possible pour Cotta Messallinus. Quant à L. Aurelius Cotta ${ }^{101}$, consul en 65 av. J.C. et censeur l'année suivante, il est fort probable de le considérer comme déjà mort, puisque lors du rappel d'exil de Cicéron, P. Lentulus Spinther lui donne la parole en premier pour s'exprimer, preuve de son ancienneté parmi les sénateurs de rang censorien ${ }^{102}$. Comme le fait remarquer R. Syme, le père adoptif pourrait être un Aurelius Cotta qui ne serait pas connu par ailleurs, peutêtre un fils du préteur de 54 av. J.-C., M. Cotta ${ }^{103}$. Force est d'admettre que l'identité du père adoptif ne peut pas être tranchée.

Un texte médiéval ${ }^{104}$, où les réalités romaines ne sont plus si familières, mentionne l'adoption de Cotta $^{105}$, mais de manière erronée : Hic ab Aurelio Cotta adoptatus M. Aurelius Maximus uocabatur. Puis, il narre l'épisode du combat singulier qui opposa Valerius à un Gaulois. Le texte explique parfaitement l'origine du cognomen de Coruinus. Néanmoins, le Romain n'est pas ici appelé Valerius, mais Aurelius Messalla. Le nomen est faux et le cognomen est apocryphe. Ces erreurs attestent la méconnaissance de l'histoire des gentes romaines les plus célèbres. Effectivement, il paraît difficile pour un Romain de confondre un Aurelius et un Valerius.

Cotta s'inscrit pleinement dans cette tradition de la gens romaine et le Principat ne modifie nullement l'importance de cette dernière. La continuité est de mise. Les prétentions généalogiques et le prestige du patriciat sont affirmés, comme sous la République, et offrent l'image d'une stabilité familiale, qui permet de se définir par rapport aux nouvelles élites, ne pouvant pas se vanter de telles origines. Néanmoins, ce n'est pas une famille figée, puisque l'adoption par un Aurelius Cotta est la preuve d'une alliance familiale avec la noblesse plébéienne et qui assure la pérennité des Aurelii Cottae. La lignée prestigieuse reste un atout considérable pour affirmer sa prééminence au sein de l'ordre sénatorial, qu'Auguste organise.

\section{LES MARQUEURS POLITIQUES DE L'IDENTITÉ NOBILIAIRE AU DÉBUT DU PRINCIPAT}

\section{La carrière}

C'est le moyen le plus évident pour appréhender ce critère politique. Les patriciens occupent une place à part car ils sont porteurs d'un imaginaire historique : leur prétention à être les descendants des premiers sénateurs de Rome leur confère une légitimité et une identité spécifiques ${ }^{106}$. Dans quelle mesure la carrière de Cotta illustre la res publica restituta voulue par Auguste? D'abord, il gravit aisément les échelons du cursus honorum, mais nous ne savons rien avant l'exercice de la préture. Toutefois, les liens étroits entre la famille des Valerii Messallae et le pouvoir impérial permettent de suggérer l'exercice des magistratures suo anno ${ }^{107}$. Sa questure pourrait être située en 12. Certains placent une participation

101. P. von Rohden, $R E$, Aurelius $\mathrm{n}^{\circ}$ 102, col. 2485-2487.

102. Cicéron, Pour Sestius, XXXIV, 73.

103. R. Syme, 1978, p. 120.

104. L'œuvre est généralement attribuée à Cornutus, contemporain de Perse. La méconnaissance de l'histoire des gentes romaines, encore connue dans l'Antiquité tardive (cf. Macrobe, Saturnales, I, 26 : Sic Messala tuus, Auiene, dictus a cognomento Valerii Maximi, qui, postquam Messanam urbem Siciliae nobilissimam, cepit, Messala cognominatus est), fait plutôt penser à une œuvre médiévale et probablement réalisée, à l'époque carolingienne, se fondant sur un matériel composé d'exégèses précédentes.

105. Commentum Cornuti in Persium in Satiram II, 71-72: Quin damus id superis, de magna quod dare lancel Non possit magni Messalae lippa propago? [...] Per Messalae autem propaginem eos uult accipi qui non pura conscientia diis sacrificant. Hic autem Cottam Messalinum dicit, qui tam uitiosos oculos in senectute habuit ut palpebrae eius in exteriorem partem uerterentur; fuit enim et multis deditus uitiis. Hic ab Aurelio Cotta adoptatus M. Aurelius Maximus uocabatur, originem trahens ab Aurelio Messala qui septies fuit consul; qui cum a quodam Gallo ad mono-

machiam in proelio uocaretur, coruus super galeam in caput eius sedit, et uicto hoste Coruinus appellatus est. "Que ne donnonsnous pas aux dieux ce que ne pourrait leur donner sur un grand plat la descendance aux yeux malades du grand Messalla? [...] Mais en utilisant la descendance de Messalla, il veut désigner ceux qui ne sacrifient pas aux dieux avec des yeux intègres. Tandis qu'il parle de ce Messallinus Cotta, qui, dans sa vieillesse eut des yeux si mauvais, dont les paupières partaient de chaque côté vers l'extérieur. Il s'adonna à de nombreux vices. Celui-ci fut adopté par Aurelius Cotta et on l'a appelé M. Aurelius Maximus. Il tirait son origine d'Aurelius Messalla qui fut sept fois consul; qui provoqua en duel singulier un certain Gaulois, un corbeau se posa sur le casque qu'il portait sur sa tête, et la victoire sur l'ennemi le fit appeler Corvinus" (traduction personnelle).

106. Dans le domaine politique, les patriciens jouissent de privilèges statutaires dès le début du Principat d'Auguste, avec notamment la mise en place progressive d'un "cursus patricien». cf. en dernier lieu R. Baudry, 2006, p. 169-171.

107. Ce point est développé p. 27-29. Concernant l'exercice des magistratures suo anno, la carrière de Cotta n'a rien d'ori- 
hypothétique à l'expédition rhénane de 12-13 sous les ordres de Tibère ${ }^{108}$. La nature de la questure n'a guère suscité l'intérêt des historiens, alors qu'un faisceau non négligeable d'indices fait pencher vers l'attribution d'un poste de quaestor Augusti. Dans sa monographie sur ces questeurs, M. Cébeillac ${ }^{109}$ mentionne les pré-requis : la parenté avec la famille impériale, le prestige et l'ancienneté de la gens, la carrière éminente d'un proche ou encore l'amitié avec l'empereur ou un de ses proches. Cotta remplit toutes ces conditions. De surcroît, la majorité des questeurs est constituée d'individus issus du patriciat et l'étude prosopographique de ces questeurs montre que, sur une petite trentaine d'individus connus sous la dynastie julio-claudienne, tous ceux dont la carrière est renseignée et n'a pas été abrégée par un suicide, une exécution ou une mort prématurée, ont été proconsuls d'Asie au sommet de leur carrière, à l'exception de L. Nonius Asprenas qui poursuivit sa carrière sous les Flaviens et obtint le proconsulat d'Afrique. Enfin, les quaestores principis et candidati de la fin du règne d'Auguste sont peu connus. Cotta pourrait ainsi être inséré en 12 pour l'exercice de sa questure, même si l'argument $a$ silentio ne fait évidemment pas office de preuve.

Quant à la préture, il l'aurait exercée en $17^{110}$. Lorsqu'il intervient au Sénat le 14 septembre 16 pour le procès de Scribonius Libo ${ }^{111}$, il pourrait être déjà préteur désigné. En effet, lors d'une année normale, les élections pour la préture se déroulent à la mi-septembre. Le quiproquo est né du fait que ce passage de Tacite mentionne d'anciens consuls et Cotta ouvrait la liste, en étant nommé Cotta Messallinus. Or il prit ce deuxième cognomen à la mort de son frère aîné, soit pas avant 21. Quoi qu'il en soit, cette préture ne semble plus être polémique.

Cotta a ensuite obtenu le consulat ordinaire en 20 avec son neveu M. Valerius Messalla, fils de M. Valerius Messallinus (cos. 3 av. J.-C.) ${ }^{112}$. Ils ont été les seuls consuls de l'année, puisqu'il n'y a pas eu de consuls suffects. Le court intervalle entre la préture et le consulat est la conséquence du statut de patricien. Il atteste aussi le prestige de la famille et les relations étroites avec le pouvoir impérial. Un sénatus-consulte est mentionné par le Digeste lors de ce consulat ${ }^{113}$. Mais la disparition de Germanicus fut l'événement de l'année et permet d'obtenir des sources sur le contenu de l'année consulaire de Cotta et son activité politique.

Les dernières décennies ont été marquées par des découvertes épigraphiques primordiales, permettant d'éclairer les conséquences de la mort de Germanicus, notamment celle de la Tabula Siarensis ${ }^{114}$, retrouvée à Siarum ${ }^{115}$. L'inscription concerne les honneurs funèbres à rendre à la mémoire de Germanicus, mort à Antioche le 10 octobre 19. Il s'agit donc d'une lex Valeria Aurelia, du nom des deux consuls de l'année 20. Toutefois, ginal, puisque de nombreux sénateurs proches du pouvoir impérial et membres de la nobilitas ont bénéficié de ce rythme de carrière.

108. C'est le cas notamment de D. Voisin, 2000, p. 414 et 420. D. Voisin s'appuie sur un passage des Pontiques et plus particulièrement sur le terme de sospes (III, 2, 3). Ainsi, si Ovide espère que Cotta reste sain et sauf, ce serait grâce à la participation à l'expédition rhénane. Néanmoins, rien ne vient étayer ces dires.

109. M. Cebeillac-Gervasoni, 1973, p. 5.

110. R. Syme, 1986, p. 236. Un fragment des Fasti Arualium mentionne des magistrats pour l'année 17. Degrassi (Inscr. It., XIII, 1, p. 297) transcrit : [------]S [...]A PER(egrinus). Or, si nous prêtons attention au dessin de l'inscription, nous distinguons les premières lettres du nom du magistrat (les moitiés d'un $\mathrm{C}$ et d'un $\mathrm{O}$, ainsi qu'une partie d'un $\mathrm{T}$ ). C'est pourquoi, R. Syme propose la transcription suivante: COT[T]A : (R. Syme, 1956, p. 18).

111. Tacite, Annales, II, 32,1.

112. Diverses sources mentionnent ce consulat: épigraphiques (comme CIL, VI, 32270), littéraires (telle Tacite, Annales, III, 2,3) et juridiques dont Digeste, I, 16, 4, 2 (De officio proconsulis et legati) Vlpianus 1 de officio proconsulis.
113. Digeste, XLVIII, 2, 12, 3 (De accusationibus et inscriptionibus) Venuleius Saturninus lib. 2 de iudiciis publicis : Si seruus reus postulabitur, eadem obseruanda sunt quae si liber esset, ex senatus consulto Cotta et Messala consulibus.

114. F. Fernández et J. González, 1981, p. 1-36 : cet article permet d'obtenir une édition du texte, une traduction en espagnol, mais surtout un commentaire, peu de temps après la découverte des fragments; c'est aussi le cas d'un autre article qui reprend très sensiblement les mêmes éléments : J. González, 1984, p. 55-100. Cette inscription est fondamentale pour comprendre l'histoire du début du I ${ }^{\text {er }} \mathrm{s}$. ap. J.C. Nous ne nous étendrons pas ici sur la portée de ce texte, afin de saisir l'idéologie impériale au début du règne de Tibère, $c f$. F. Millar, 1988, p. 11-19. La Tabula Siarensis a fait l'objet d'une bibliographie très conséquente. Nous renvoyons à M. H. Crawford (dir.), 1996, plus particulièrement p. 507-547 et A. Fraschetti (dir.), 2000. Elle doit être rapprochée de la Tabula Hebana, puisque les six premières lignes de celle-ci se retrouvent dans les neuf dernières lignes de la Tabula Siarensis.

115. Cependant, malgré le titre donné à l'inscription, il n'est pas certain qu'elle provienne de Siarum, cf. J. González, 1984, p. 82-87 et notamment P. Le Roux, 1988, p. 23-26. 
cette inscription ne date pas de l'année 20, puisque Valerius Messalla et Aurelius Cotta sont consules designati, c'est-à-dire qu'ils ont été élus, mais ne sont pas encore entrés en charge. De facto, les événements relatés se placent entre le 10 octobre 19 (en sachant que la nouvelle de la mort de Germanicus n'est pas parvenue tout de suite à Rome) et le 31 décembre de la même année.

Les lignes 27-31 ${ }^{116}$ sont importantes pour comprendre le rôle du Sénat au début de l'époque impériale et elles permettent de compléter le passage de Tacite sur les honneurs rendus à Germanicus ${ }^{117}$. Effectivement, ce passage n'explique pas qui prend la décision finale à travers le vote. Or, d'après cette inscription, nous savons qu'il s'agit du Sénat. L'inscription indique quelque chose de totalement nouveau : ce vote a été suivi par une loi présentée au peuple par les consuls, Cotta et Messalla. F. Millar propose néanmoins une autre explication ${ }^{118}$. Le Sénat ne pourrait prendre des décisions que s'il était assuré $\mathrm{du}$ consentement du peuple. Nous n'entrerons pas dans ces querelles historiographiques, faute d'élément nouveau.

Une autre trouvaille vient éclairer les relations entre Cotta, le Sénat et l'empereur. En effet, le sénatus-consulte de $\mathrm{Cn}$. Pisone patre a permis de connaître en détails le procès de Pison et ses conséquences ${ }^{119}$. Sans tenter de résumer toutes les études consacrées à ce sénatus-consulte, nous nous limiterons aux éléments liés à Cotta. Notons que son frère intervient durant les débats en tant que premier consulaire ${ }^{120}$ cité et prenant part au comité de rédaction du sénatus-consulte. Quant à

116. Fragment II colonne b 1. 27-31 : [...] Vtique M(arcus) Messalla M(arcus) Aurelius / Cotta Maximus co(n)s(ules) designati cum magistratum inissent primo quoque tempore cum per / auspicia liceret sine binum trinumue nundinum prodictione legem ad populum de I honoribus Germanici Caesaris ferendam curent. Cens (uere). I(n) s(enatu) f(uerunt) CCLXXXV.H(oc) s(enatus) c(onsultum) per relatio/nem secundam factum est unum.

117. Tacite, Annales, II, 83. Tacite s'appuyait beaucoup sur les Acta senatus. On pouvait alors aisément penser qu'il s'agissait d'un vote du sénat mais nous n'en avions jusqu'alors pas la preuve.

118. F. Millar, 1988, p. 13

119. Deux éditions virent le jour à quelques mois d'intervalle et avec quelques différences sensibles : A. Caballos, W. Eck et F. Fernández, 1996 et W. Eck, A. Caballos et F. Fernández, 1996. Nous disposons de tables entières de bronze et de plusieurs fragments. D'origines diverses, ces découvertes correspondent à huit copies conservées du même sénatus-
Cotta, il est uniquement mentionné en tant que consul de l'année avec son neveu $M$. Valerius Messalla à la ligne 175 de la copie A (Cotta et Messalla) ${ }^{121}$; la copie B n'a conservé que [...] essala cos. (ligne 126).

Citer Cotta uniquement à propos de la subscriptio de Tibère ne laisse pas transparaître le rôle important qu'il a joué lors des débats. En tant que consul ordinaire, Cotta a exprimé la prima sententia. Le récit de Tacite met en lumière le rôle joué par l'aristocrate : Primus sententiam rogatus Aurelius Cotta consul (nam referente Caesare magistratus eo etiam munere fungebantur) nomen Pisonis radendum fastis censuit, partem bonorum publicandam, pars ut Cn. Pisoni filio concederetur isque praenomen mutaret; $M$. Piso exuta dignitate et accepto quinquagies sestertio in decem annos relegaretur, concessa Plancinae incolumitate ob preces Augustae ${ }^{122}$. Nul doute que la mention de la pietas et de la seueritas des juges ${ }^{123}$ fait allusion à l'intervention du consul Cotta, puisque Tacite fondait son travail sur les acta senatus et il a dû avoir accès aux documents liés à ce sénatus-consulte. En tant que consul, Cotta aurait dû présider la séance du Sénat, mais compte tenu de l'ordre du jour exceptionnel, c'est Tibère en personne qui préside, rappelant son auctoritas supérieure. Les sénateurs ayant exposé leurs sententiae sont tous des proches du régime impérial. Par ailleurs, les lignes 71 à 123 se fondent en grande partie sur les propositions de Cotta citées par Tacite. La disparition des fastes est une damnatio memoriae. Le sénatus-consulte final n'est pas allé si loin, mais le nom de Pison a été martelé sur une statue de Germanicus, élevée par les sodales

consulte. La copie A est la plus complète (quelques petits fragments manquants) et elle provient du lieu où fut trouvée la loi d'Irni.

120. Il est visible dès la praescriptio à la ligne 2 : Adfuerunt M. Valerius $M . f$. Lem. Messallinus (copie A).

121. Les deux consuls sont uniquement nommés par leurs cognomina.

122. Tacite, Annales, III, 17, 4 : «Invité le premier à donner son avis, le consul Aurelius Cotta - car, lorsque le rapport était fait par César, les magistrats s'acquittaient aussi de cette charge - proposa de rayer des fastes le nom de Pison et de confisquer une partie de ses biens, en accordant l'autre à son fils, Cn. Piso, qui changerait de prénom; M. Piso, dépouillé de sa dignité, mais recevant cinq millions de sesterces, serait relégué pour dix ans, alors qu'on accorderait à Plancine la vie sauve grâce aux prières d'Augusta» (Traduction P. Wuilleumier, C.U.F.)

123. Ligne 172 (copie A). 
Augustales $^{124}$. Tibère a refusé que le nom de Pison soit retiré des fastes. Cependant, il l'est de certaines inscriptions ${ }^{125}$. La proposition de la confiscation des biens et surtout celle d'en transférer une partie au fils de Pison ont en revanche été suivies $^{126}$. Toutefois le sénatus-consulte diffère quelque peu du récit de Tacite. En effet, le premier maintient la somme de cinq millions de sesterces répartie en une dos d'un million et un peculium de quatre millions. En se suicidant, Pison avait pu sauver une partie de ses biens. Ce n'est pas le fils de Pison qui en est le bénéficiaire, mais Calpurnia, non mentionnée par Tacite. Cn. Pison fils a bien changé de praenomen, s'appelant Lucius après le sénatus-consulte. Quant au sort réservé à Plancine dans le sénatus-consulte, il fait écho aux préconisations de Cotta. Elle a obtenu le soutien éminent de Julia Augusta ${ }^{127}$ et a été graciée ${ }^{128}$. Finalement, seul $\mathrm{Cn}$. Pison père est condamné et le reste de la famille est épargné.

Le prestige d'une gens se mesure aussi aux promagistratures exercées. Après son consulat, Cotta a exercé un proconsulat en Asie $^{129}$. R. Syme ${ }^{130}$ n'hésite pas à souligner que ce départ en Asie a dû être un soulagement pour le Sénat et pour la société romaine... Ce jugement de valeur est repris de la tradition tacitéenne dont nous serons amenés à traiter. La datation de ce proconsulat est

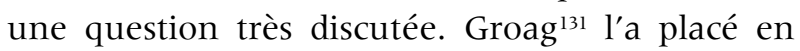
$22 / 23,25 / 26$ ou 27/28. Cette datation basse s'explique par le fait que Groag pensait qu'il y avait deux Cotta. Bien que Degrassi ${ }^{132}$ ait accepté la reconnaissance d'un seul Cotta, il a repris les propositions de Groag, même s'il écarte la première. Selon lui, si l'intervalle de cinq ans est maintenu, les deux autres dates proposées par Groag sont envisageables. Néanmoins, la période $26 / 28$ ne peut pas être retenue car elle correspond au biennum de M. Aemilius Lepidus. Selon V. Chapot,

124. Lignes 82-83 (copie A)

125. C'est le cas de Dessau, 95.

126. Lignes 93-103.

127. Ligne 115.

128. Lignes 112-113.

129. Voir infra p. 31-34 pour les relations clientélaires en Orient de Cotta, en particulier pour la province d'Asie.

130. R. Syme, 1986, p. 237.

131. E. Groag, $P I R^{2}, 1933, \mathrm{n}^{\circ} 1488$.

132. A. Degrassi, 1946, p. 38.

133. V. Chapot, 1941, p. 89. Selon V. Chapot, cette date pourrait la datation de $36 / 37$ pourrait convenir ${ }^{133}$. R. Syme préfère, quant à lui, avancer la date de 35/36 et Cotta aurait succédé à P. Petronius $(\cos .19)^{134}$. Cette dernière date est dorénavant communément admise ${ }^{135}$. Après le proconsulat d'Asie, nous perdons la trace de Cotta.

La carrière politique de Cotta est celle d'un homme politique doté de la faveur impériale et elle est caractéristique d'un patricien, suivant un cursus particulier sous le Principat. Cependant, la valeur d'un sénateur se définit également par ses discours et ses prises de position lors des débats sénatoriaux et Cotta ne déroge pas à la règle.

\section{Les interventions au Sénat}

D'abord, elles ne sont pas empreintes de moderatio, une des vertus capitales de la noblesse romaine ${ }^{136}$. Lorsqu'il s'est exprimé en faveur de la damnatio memoriae de Libo $^{137}$ en 16, il a montré, dès sa première prise de parole mentionnée par Tacite, son intransigeance. Ce dernier cite, entre autres, cette sentence, afin de mettre en exergue l'ancienneté de l'adulatio ${ }^{138}$, donnant une vision péjorative de la nobilitas impériale. Les séances sénatoriales sont un moment privilégié pour jauger l'adhésion des sénateurs au dessein impérial. Ainsi, Fr. Hurlet a montré que le pouvoir impérial, au I ${ }^{\text {er }}$ s., était "visible et accessible» ${ }^{139}$. Cotta exprime son allégeance lors des débats et l'adulatio principis «doit être analysée comme la forme exacerbée d'un nouveau cérémonial propre au Sénat d'époque impériale " ${ }^{140}$. Ensuite, il faut attendre l'année du consulat de Cotta pour le retrouver dans le récit de Tacite. C'est à nouveau dans le cadre d'un procès, cette fois pour condamner la mémoire de Cn. Pison ${ }^{141}$. Puis en 24, il intervient au Sénat, afin de proposer que les gouverneurs soient tenus pour responsables des actes de leurs épouses ${ }^{142}$, alors que son frère, Messallinus, s'était insurgé en 21

aussi convenir à P. Cornelius Lentulus Scipio.

134. R. Syme, 1986, p. 238.

135. U. Vogel-Weidemann, 1982, p. 291-292.

136. Chr. Badel, 2005, p. 169.

137. Tacite, Annales, II, 32, 1.

138. Ibid., II, 32, 2.

139. Fr. Hurlet, 2000, p. 131.

140. Ibid., p. 136.

141. Tacite, Annales, III, 17, 4.

142. Ibid., IV, 20, 4 
contre une mesure similaire souhaitée par Caecina $^{143}$. La province est avant tout la sphère traditionnelle des opérations militaires où le gouverneur exerce pleinement son imperium ${ }^{144}$. Ce discours est aussi un épilogue à la persécution de Sosia Galla, épouse de C. Silius, légat de Germanie supérieure. Sosia était une amie d'Agrippine l'aînée, la veuve de Germanicus, et elle fut exilée après avoir été accusée de trahison avec son mari ${ }^{145}$. Comme le fait remarquer R. Syme, Cotta pourrait ainsi mener une vengeance contre la famille de Germanicus, afin de plaire à Tibère ${ }^{146}$. En outre, le Digeste $^{147}$ cite une loi, datant du consulat de Cotta en 20, stipulant que les gouverneurs devaient être punis pour les délits commis par leurs épouses. C'est certainement une erreur de date. La confusion était compréhensible, puisque Cotta était l'instigateur de cette mesure. Ulpien a donc cru qu'elle datait de son consulat et il a substitué Cotta et Messalla consulibus à Cethego et Varrone consulibus ${ }^{148}$. En 29, Cotta intervient lors des débats contre Agrippine l'aînée et Nero. La teneur de ses propos n'est pas rapportée par Tacite qui se borne à préciser le sens de l'intervention : promptissimo Cotta Messalino cum atroci sententia ${ }^{149}$. Nous apprenons un peu plus loin qu'il s'agit de la peine capitale. Mais cette proposition tourne court, puisque les magistrats, c'est-à-dire les consuls n'osent pas prendre cette décision. Enfin, notre connaissance de Cotta est très largement tributaire du récit de Tacite et les lacunes des Annales entraînent une méconnaissance complète de certains aspects de sa carrière, par exemple au moment de la disgrâce de Séjan.

Les prises de position du fils de Corvinus étaient tranchées et ne suscitaient pas le consensus de ses pairs. Ses détracteurs stigmatisaient la démesure des propositions et son style de vie outrancier ${ }^{150}$. Cette attitude est à rapprocher du nouveau positionnement des sénateurs sous le

143. Ibid., III, 34, 2-5. Pour de plus amples renseignements, cf. A. J. Marshall, 1975, p. 109-128.

144. A. J. Marshall, 1975, p. 110

145. Tacite, Annales, IV, 18-19.

146. R. Syme, 1978, p. 130.

147. Digeste, I, 16, 4, 2 (De officio proconsulis et legati) Vlpianus 1 de officio proconsulis : Proficisci autem proconsulem melius quidem est sine uxore : sed et cum uxore potest; dummodo sciat senatum, Cotta et Messala consulibus, censuisse futurum, ut si quid uxores eorum qui ad officia proficiscuntur deliquerint, ab ipsis ratio et uindicta exigatur. règne de Tibère. En effet, celui-ci stigmatisait les dépenses luxueuses effectuées par des nobiles ${ }^{151}$. De nombreux sénateurs suivirent le Princeps. En outre, les mentalités sénatoriales n'étaient pas homogènes sur ce point. Tacite oppose les membres de la nobilitas romaine, adeptes de la magnificentia aux nouveaux sénateurs issus des élites italiennes et provinciales qui ont gardé leur domestica parsimonia ${ }^{152}$.

\section{Le procès}

Cotta a été accusé par d'autres sénateurs, parmi lesquels seul le nom de Caesilianus est conservé $^{153}$. Le livre VI des Annales est consacré à toute une série de procès pour maiestas. Cotta a été poursuivi en 32 , selon ce chef d'accusation, pour avoir fait des plaisanteries dérisoires sur Livie et sur la virilité douteuse de Caligula. L'accusation de maiestate inclut les offenses, verbales ou non, contre la personne du Prince, son prédécesseur et sa famille. Comme le fait remarquer B. Levick, la multiplication de procès, après la chute de Séjan, serait due à Caligula et à ses proches, notamment les procédures concernant les attaques personnelles contre Caligula ${ }^{154}$. C'est le cas de Cotta. La coterie de C. César était hostile au noble qui affichait trop clairement son allégeance inconditionnelle à Tibère.

Cotta ne fut pas condamné, grâce à une lettre de soutien de l'empereur écrite lors de sa retraite à Capri et lue au Sénat. Tibère retrace l'origine de leur amitié, la fidélité du sénateur et les services rendus ${ }^{155}$. Puis, Tacite rapporte d'autres passages de la lettre, en les citant précisément ${ }^{156}$. Selon R. S. Rogers, ce serait une marque d'irritation et d'impatience, tandis que B. Levick pense qu'il s'agit d'un jeu avec des expressions prises chez des poètes comiques ${ }^{157}$. L'ancienneté de l'amitié n'était pas un gage d'acquittement à la fin du Prin-

148. L. Fanizza, 1977, p. 208-209.

149. Tacite, Annales, V, 3, 2.

150. Ibid., VI, 5, 1 .

151. Ibid., III, 52, 3.

152. Ibid., III, 55, 4.

153. Ibid., VI, 5, 1. Y. Rivière, 2002, p. 513 n 13.

154. B. Levick, 1976, p. 205

155. Tacite, Annales, VI, 5, 2.

156. Ibid., VI, 6, 1.

157. R. S. Rogers, 1933, p. 122-123 et B. Levick, 1976, p. 201 
cipat de Tibère. En effet, certains amis de longue date ont été condamnés, tel Vistilius ${ }^{158}$. Après l'acquittement de Cotta et l'exil du principal délateur Caesilianus, Tacite en profite pour signaler que l'aristocrate était ruiné par son luxe ${ }^{159}$. Revenons en dernier lieu sur l'identité du délateur ${ }^{160}$. Son nom est très souvent orthographié Caecilianus, en se fondant sur l'existence du préteur de 21, Magius Caecilianus ${ }^{161}$, alors que la forme Caesilianus est attestée ${ }^{162}$. Pourquoi est-ce que cet homme a accusé Cotta? Quel était l'intérêt? Y. Rivière ${ }^{163}$ a démontré que si le procès était remporté, le délateur obtenait une certaine potentia facilitant grandement la poursuite de sa carrière sénatoriale. C'est d'autant plus vrai que Caesilianus aurait une origine modeste, même s'il s'agit d'un sénateur, hypothèse corroborée par le fait que nous ne savons rien de sa carrière politique et qu'il fut exilé à l'issue du procès.

La carrière de Cotta s'est ancrée dans le domaine politique. Contrairement à son père honoré d'un triomphe ${ }^{164}$, ou à son frère ayant reçu les ornements triomphaux ${ }^{165}$, Cotta ne semble pas avoir eu une carrière militaire brillante. Plusieurs hypothèses peuvent être avancées. D'abord cet aspect peut être mis en corrélation avec le désintérêt progressif de la nobilitas romaine pour les faits d'armes, même si nous ne sommes que dans la première moitié du ${ }^{\text {er }}$ s. ap. J.-C. ${ }^{166}$. Ensuite, son tempérament pouvait le rendre peu digne de confiance. Enfin, la documentation n'est peut-être pas parvenue jusqu'à nous.

\section{L'exercice des sacerdoces}

Cotta a probablement obtenu deux prêtrises contribuant à sa dignitas et à sa prééminence sociale. Le premier sacerdoce connu est celui des saliens palatins, dont tous les membres sont des

158. Tacite, Annales, VI, 9, 2

159. Ibid., VI, 7, 1.

160. Sur ce point, nous renvoyons à l'article de R. Syme (1949, p. 10) et à Y. Rivière, 2002, p. 513 no 13.

161. Tacite, Annales, III, 37, 1

162. IG, XIV, 1584.

163. Y. Rivière, 2002, p. 412-413.

164. Tibulle, Élégies, II, 1, 33

165. Velleius Paterculus, Histoire romaine, II, 112, 2.

166. Chr. Badel, 2005, p. 175-177.

167. Comme l'inscription provient d'un marchand d'antiquités, nous ne disposons d'aucune information sur son origine. Toutefois, une provenance romaine paraît la plus probable, patriciens. C'est notamment pour préserver ces anciennes prêtrises qu'Auguste a créé de nouveaux patriciens et cet acte politique participe pleinement à la continuité du patriciat entre la République et le Principat. Une inscription ${ }^{167}$ présente une liste de personnages issus des grandes gentes romaines et des membres de la famille impériale. Il s'agit des fastes d'un collège sacerdotal. Est-il possible de l'identifier? Le plus simple est de procéder par élimination. Ainsi, il faut éliminer le collège pontifical, puisque les noms ne sont pas ordonnés en decuriae, de même que celui des augures et des sodales augustales. Le formulaire de cooptation des frères arvales est radicalement différent, ce qui exclut aussi ce collège. En comparant avec d'autres fragments, il est possible d'identifier cette liste à celle du collège des salii palatini. D'après l'étude d'autres fragments relatifs à ce collège, l'inscription devrait présenter la date consulaire de l'année, puis une série de noms au nominatif, qui fait référence aux membres cooptés dans l'année, chacun suivi de in locum et le nom de la personne remplacée. Un autre collège utilise ce type de formules, celui des salii collini, mais la présence de membres de la famille impériale permet d'opter pour le premier, plus prestigieux. Enfin, il reste à déterminer la date de ce fragment. Ce dernier pourrait être antérieur aux années 8-11 ap. J.-C. ${ }^{168}$ et vraisemblablement dater de $4^{169}$. Cotta aurait donc été coopté vers l'âge de 18 ans, ce qui n'est pas rare dans ce collège.

Quant à la seconde prêtrise dont fait mention Tacite, elle est difficilement identifiable : Cum die natali Augustae inter sacerdotes epularetur, nouendialem eam cenam dixisse ${ }^{170}$. Aucun indice n'est fourni pour reconnaître ce sacerdoce. Cependant, nous pouvons en rejeter certaines. Tout d'abord, son frère, Messallinus, était frère arvale et quindecemuir sacris faciundis ${ }^{171}$. Ce serait peu probable

cf. C. Letta, 2000, p. 521-537 et surtout p. 521-524. Nous reprenons ici l'essentiel de sa démonstration.

168. Pour une démonstration mettant en lumière les indices chronologiques, cf. C. Letta, 2000, p. 525-528.

169. C. Letta, 2000, p. 537 : année de l'adoption de Tibère par Auguste. Car Drusus étant pontife en 8 , il est fort probable qu'il ait obtenu une charge sacerdotale moins prestigieuse auparavant.

170. Tacite, Annales, VI, 5, 1.

171. Tibulle, Élégies, II, 5. Cette élégie fut écrite à l'occasion de l'entrée de Messallinus dans le collège religieux des Quindecemuiri. Il est également mentionné lors des Jeux Séculaires de 17 av. J.-C. (CIL, VI, 32323). 
d'avoir deux frères occupant la même charge. Messalla Corvinus était augure ${ }^{172}$. Cotta aurait ainsi très bien pu occuper la place laissée vacante après la mort de Corvinus. Ce sacerdoce ne fait certainement pas allusion à celui de salien car certaines charges, comme la préture et le consulat, sont inconciliables avec cette prêtrise et obligent à démissionner par l'acte d'exauguratio ${ }^{173}$. Or, en 32 , Cotta est consulaire et ne peut plus faire partie de ce collège depuis longtemps. Elle ne peut pas s'ajouter à celle de pontife et d'augure ${ }^{174}$.

Les sacerdoces archaïques sont un aspect fondamental de l'identité patricienne, puisqu'ils leur sont réservés. La restauration religieuse menée par Auguste a donc permis aux patriciens de réaffirmer leur singularité. Cependant, le Princeps a créé des patriciens, notamment pour ces sacerdoces archaïques et les anciens et les nouveaux patriciens se côtoient. En nommer un dont l'ascendance remonte à la Rome des premiers siècles permet de légitimer cette restauration religieuse.

Cotta n'est pas seulement un membre de l'élite dirigeante, dont l'identité se définit par des critères classiques, déjà en vigueur sous la République, tels les magistratures et les sacerdoces. La nobilitas doit dorénavant se définir par rapport à un nouvel acteur, le Princeps.

\section{COTTA MESSALLINUS, L'AMICUS PRINCIPIS}

$C^{\prime}$ est un amicus de Tibère ${ }^{175}$ et un membre de la cour impériale ${ }^{176}$. Cette amicitia est institutionnalisée et elle permet à Cotta d'intervenir au Sénat, souvent dans les premières personnes donnant leur avis ${ }^{177}$, même avant d'être consulaire ${ }^{178}$. Les relations entre Tibère et les Valerii Messallae semblent anciennes, datant du Principat d'Auguste. En effet, Tibère a été influencé par l'élo-

172. Dion Cassius, Histoire romaine, XLIX, 16, 1. Mais la cooptation n'a pas eu lieu juste après la guerre de Sicile, comme l'indique Dion Cassius.

173. CIL, VI, 1978; Dessau, 5024.

174. J. Rüpke pensait que Cotta aurait pu succéder à son père Corvinus en tant qu'augure $\left(2005, \mathrm{n}^{\circ} 827\right.$, p. 802-803; 2007, p. 36).

175. Dans son étude prosopographique des amici principis, J. Crook mentionne Aurelius Cotta $\left(\mathrm{n}^{\circ} 49\right)$ : J. Crook, 1955, p. 153.

176. Nous n'utilisons pas le terme d'aula Caesaris qui serait ici anachronique, puisque sa première attestation date du début du principat de Claude. Certes, le terme d'aula est utilisé par Tacite, mais c'est parce qu'il emploie la formulation de son quence de M. Valerius Messalla Corvinus, le père de Cotta, et l'a pris comme modèle ${ }^{179}$. En outre, une épître d'Ovide rapporte l'annonce par Cotta de la mort d'un dénommé Celsus ${ }^{180}$. R. Syme a voulu reconnaître en lui Celsus Albinovanus, scriba et comes en 20 av. J.-C. de Tibère ${ }^{181}$. Ovide a probablement dû connaître Tibère dès cette époque, puisque Celsus a publié les Amours du poète. Messalla Corvinus aurait ainsi pu faire la connaissance de Tibère à ce moment.

Cotta fait donc partie de la cour de l'empereur, c'est-à-dire de son entourage proche. Les membres sont de statuts extrêmement divers et la composition de cet entourage ne fait pas l'objet d'un consensus. D'après A. Winterling, la cour impériale julio-claudienne serait constituée majoritairement de chevaliers et d'affranchis, son «aristocratisation", perceptible à l'époque flavienne ne serait pleinement accomplie que sous les Antonins $^{182}$. Il cite les exemples de Mécène ou de Séjan. Or, les membres des anciennes familles sénatoriales républicaines étaient aussi présents ${ }^{183}$. Cotta faisait partie de la cour impériale. Ce n'est pas le seul cas attesté au sein de la gens des Valerii, puisque son frère, Messallinus, était est un proche d'Auguste et fut un membre du conseil impérial ${ }^{184}$.

En dépit des liens étroits existants entre Tibère et Cotta, il ne semble pas y avoir eu d'alliance matrimoniale les rapprochant directement. Cependant, des liens matrimoniaux avaient déjà été tissés entre les Valerii Messallae et la famille impériale. En effet, M. Valerius Messalla Barbatus Appianus (cos. 12 av. J.-C.) avait épousé Marcella Minor et son fils était marié à Domitia Lepida. De cette dernière union naquit Messaline, l'épouse de Claude $^{185}$. Son respect de la famille impériale est tel que Cotta envoie des statuettes de Tibère, Auguste et Livie à Ovide exilé à Tomes ${ }^{186}$.

époque pour une réalité plus ancienne. Sur ce point, voir : A. Winterling, 1999, p. 195-203 et Fr. Hurlet, 2001, p. 182. 177. Fr. Hurlet, 2000, p. 144.

178. Tacite, Annales, II, 32, 1.

179. Suétone, Tibère, LXX, 1.

180. Ovide, Pontiques, I, 9, 1.

181. R. Syme, 1978, p. 90.

182. A. Winterling, 1999, p. 161-194.

183. Fr. Hurlet (2001, p. 175) a montré l'influence des sénateurs, à l'époque julio-claudienne, au sein de la cour impériale.

184. Papyrus d'Oxyrhynchos, 2435. Messallinus a occupé une place de premier plan également sous le principat de Tibère. 185. Suétone, Claude, XXVI, 2. FOS, $\mathrm{n}^{\circ} 774$.

186. Ovide, Pontiques, II, 8, 1-4. 
Les liens tissés entre Tibère et Cotta sont une des manifestations de l'adaptation de la noblesse républicaine à la nouvelle donne politique du Principat, mais il ne faut pas nier la profonde amitié entre les deux personnages. Cotta n'hésitait pas à appeler l'empereur Tiberiolus meus ${ }^{187}$. Cette proximité lui a permis d'être protégé lors de son procès, en 32, après la disgrâce de Séjan. Tacite en profite pour dresser un portrait sévère de Cotta. L'amitié avec Tibère est stigmatisée, présentée comme une forme d'adulation servile.

Le rôle politique inscrit pleinement Cotta dans cette tradition aristocratique héritée de l'ère républicaine, mais ses liens personnels avec Tibère l'ont clairement rangé dans la sphère des amici principis de premier plan. Néanmoins, l'amitié avec l'empereur n'explique pas à elle seule la prééminence de l'aristocrate. Ce dernier se définit également par son assise sociale et ses réseaux clientélaires.

\section{LA CLIENTÈLE ET LE PATRONAT,}

\section{MARQUEURS SOCIAUX DE LA NOBLESSE}

La première occurrence est liée à la poésie funéraire. Une inscription sur marbre a été retrouvée, en 1864, près de la Via Appia, dans l'ager Albanus $^{188}$. Celle-ci atteste l'affection que portait Cotta au libertus Zosimus. Les épitaphes d'affranchis sont nombreuses et elles témoignent des liens existants entre l'ancien maître et l'affranchi. Ces liens étroits sont renforcés par l'onomastique du fils de Zosimus avec un cognomen, dérivé de celui de son patron. Selon O. Salomies, il s'agit de la

187. Tacite, Annales, VI, 6, 1

188. Bücheler, 990; CIL, XIV, 2298; Dessau, 1949. M(arcus) Aurelius Cottae / Maximi l(ibertus) Zosimus / accensus patroni / libertinus eram, fateor, / sed facta legetur / patrono Cotta nobilis umbra mea, / qui mihi saepe libens census donauit / equestris, qui iussit natos / tollere quos aleret, / quique suas commisit opes / mihi semper et idem dotauit / natas ut pater ipse meas, / Cottanumque meum produxit / honore tribuni, quem fortis / castris Caesaris emeruit. / Quid non Cotta dedit? Qui nunc / et carmina tristis haec dedit / in tumulo conspicienda meo. I Aurelia Saturnina Zosimi. "Marcus Aurelius Zosimus, affranchi de Cotta Maximus, appariteur auprès de son patron.

J'étais un affranchi, je le concède; mais le lecteur saura l'ennoblissement de mon ombre parce que j'ai eu Cotta pour patron. C'est lui qui m'a, de bon cour, gratifié plusieurs fois d'une fortune égale à celle d'un chevalier; lui qui m'a autorisé à reconnaître mes enfants, pour pouvoir les élever; lui qui m'a toujours confié l'administration de ses richesses et a aussi doté mes filles comme un véritable père; il a fait obtenir à mon petit Cottanus le grade de tribun [légionnaire], grade dans lequel Cottanus a servi avec bravoure seule attestation du cognomen Cottanus ${ }^{189}$. Cependant, cette inscription est bien plus importante. Elle met en lumière le rôle de la nobilitas dans la promotion de nouvelles élites. Effectivement, Cotta a doté Zosimus d'une fortune plusieurs fois supérieure à la fortune minimale d'un chevalier. L'affranchi ne peut évidemment pas être promu dans l'ordre équestre. C'est pourquoi Cotta a veillé à ce que le fils de l'affranchi, Cottanus, puisse intégrer l'élite équestre ${ }^{190}$. Cette promotion sociale est originale, puisque $S$. Demougin a montré que sur 773 cas de chevaliers, seuls 9 étaient des fils d'affranchis ${ }^{191}$. Ensuite, l'épitaphe mentionne la recommandation privée ${ }^{192}$ qui a favorisé le recrutement en tant que tribun militaire ${ }^{193}$. L'armée apparaît ici comme un instrument de promotion sociale $^{194}$ et il fallait avoir un patron proche des cercles du pouvoir, afin d'obtenir une place si convoitée. Enfin, l'inscription renseigne sur le processus d'acquisition de la fortune, par le biais des dots destinées aux filles de l'affranchi qui ont pu leur permettre de faire un bon mariage et de s'élever socialement, et par le biais des dons pour asseoir la fortune de l'affranchi et celle de son fils. Ces dons augmentent aussi le prestige du bienfaiteur ${ }^{195}$ car la liberalitas est une des uirtutes nobiliaires ${ }^{196}$

L'épitaphe versifiée, composée de cinq distiques, a suscité l'intérêt des spécialistes des carmina epigraphica latina ${ }^{197}$. L'élégance de ces vers pourrait indiquer qu'il s'agit de l'œuvre d'un lettré et donc très probablement de Cotta, comme le suggère l'inscription ${ }^{198}$, ami du poète Ovide, même

l'armée de César. Que Cotta ne nous a-t-il point donné? C'est lui qui, en proie au chagrin, a présentement donné ces vers pour qu'on puisse les voir sur mon tombeau. Aurelia Saturnina, [femme de] Zosimus» (traduction personnelle).

189. O. Salomies, 2008, p. 86.

190. S. Demougin, 1992, n 232.

191. S. Demougin, 1988 , p. 654 . Cet ordre de grandeur ne tient pas compte des chevaliers identifiés depuis.

192. Sur ce type de recommandation, consulter S. Demougin, 1988, p. 304-305.

193. Contrairement à certains (voir notamment, Z. Popova, 1968, p. 60), nous ne pensons pas qu'il fut tribun des cohortes prétoriennes.

194. Sur le terme d'honores, introduit dans l'armée, voir S. Demougin, 1988, p. 283.

195. S. Demougin, 1988, p. 81.

196. Chr. Badel, 2005, p. 169-170.

197. C'est le cas notamment de E. Galletier, 1922. Cette poésie versifiée est très prisée au ${ }^{\text {er }} \mathrm{s}$. grâce, entre autres, à l'élégie romaine : Z. Popova, 1968, p. 57.

198. E. Galletier, 1922, p. 154. 
s'il faut rester très prudent avec ce type d'argument. L'épitaphe fait même référence à Tibulle à travers cette expression: Haec dedit / in tumulo conspicienda $m e^{199}$. Les références au poète élégiaque sont très limitées dans ces épitaphes versifiées, mais la présence de l'auteur dans le cercle littéraire du père de Cotta a pu inciter ce dernier à imiter Tibulle. Il est possible de dater cette inscription. Le noble est appelé Cotta Maximus. Or, nous savons qu'il prit le surnom de Messallinus à la mort de son frère, peu après 21 ap. J.-C. ${ }^{200}$. L'épitaphe aurait donc été rédigée peu avant cette date. Ovide qualifie Cotta de iuuenis en $12^{201}$ et en $17^{202}$. L'âge mûr dont il est question fait penser à une datation entre 17 et $21^{203}$.

Les liens de clientèle dépassent bien évidemment la sphère privée et strictement romaine. $C^{\prime}$ est un instrument permettant d'asseoir le pouvoir et la position sociale de la noblesse impériale. Néanmoins, son importance est largement moindre que sous la République. Les relations entretenues avec les cités d'Asie sont importantes à analyser, même si, contrairement à de nombreux contemporains, les liens semblent plutôt d'ordre privé. La documentation épigraphique éclaire cet aspect grâce à plusieurs inscriptions.

Une inscription honorifique, sur une base de statue, en marbre, a été retrouvée à Ephèse en Ionie ${ }^{204}$, commanditée par Alexandros Memnonos. M. Aurelius Cotta Maximus Messallinus est honoré en tant que proconsul de la province d'Asie et en qualité d'évergète. Une autre inscription $^{205}$ a été retrouvée à Sardes. Certaines références demeurent obscures, d'autant plus que l'inscription originale est désormais perdue et était déjà lacunaire lors de la découverte. La dédicace a été commanditée par un proche du patricien, Epicharmos. Ce dernier est valorisé en tant qu'évergète. Toutefois, ces deux inscriptions, à caractère privé, ne renseignent nullement sur les actes effectués par Cotta et qui auraient pu justifier son qualificatif d'évergète. Ainsi, nous ne savons pas s'ils s'inscrivent dans le cadre strict de son proconsulat, plausible pour l'inscription d'Ephèse, ou d'un patronat de cité. Les Valerii Messallae ont tissé des liens étroits avec l'Orient romain et, particulièrement, en Asie mineure. En effet, le père de Cotta, Messalla Corvinus, est honoré à Ephèse et explicitement reconnu comme patron et évergète du sanctuaire d'Artémis et de la cité206. De même, Potitus Messalla (cos. 29 av. J.-C.) est honoré dans différentes cités en tant que patron et évergète, notamment à Milet où son rang de proconsul est rappelé ${ }^{207}$. Cotta a certainement bénéficié de la renommée de sa gens qui a donné deux proconsuls d'Asie sous le Principat d'Auguste, Potitus Messalla et Messalla Volesus ${ }^{208}$. Le patronat du père de Cotta, à Ephèse, a peut-être contribué à faire entrer la famille d'Alexandros Memnonos dans la clientèle de la gens et Cotta aurait ainsi entretenu des liens privilégiés avec Éphèse.

Enfin, une dernière inscription ${ }^{209}$ doit retenir notre attention. Il s'agit d'une dédicace impériale, figurant sur une base de statue de marbre et provenant de Ballica, à proximité d'Apollonis de Lydie. Le noble nommé ici M. Aurelius Cotta Maximus Messallinus, veille sur une construction ${ }^{210}$, financée sur ses fonds propres et conformément au souhait de M. Aelius Atticus, ami de Cotta, inconnu par ailleurs. Cette dédicace est importante, car elle atteste les liens existants entre un sénateur romain et un citoyen romain de Lydie. L'intérêt réside aussi dans la datation et nous avons un élément déterminant à la ligne 3 :

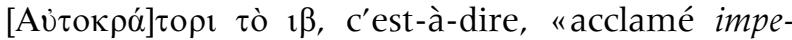
rator pour la douzième fois». La chronologie de Cotta exclut Auguste; Tibère n'a obtenu que huit salutations impériales et Caligula aucune. En fonction de cette acclamation impériale, nous pouvons
199. Tibulle, Élégies, II, 5, 19 : Haec dedit xneae sortes postquam ille parentum et II, 3, 52 : Incedat donis / conspicienda meis. Cette comparaison fut proposée par Z. Popova, 1968, p. 58-59. Cette fin de vers ne se rencontre nulle part ailleurs dans la littérature latine. L'auteur propose alors une étude littéraire afin de comparer les deux passages. Le deuxième livre des Élégies de Tibulle aurait ainsi eu une influence précoce sur la poésie funéraire.

200. Velleius Paterculus, Histoire romaine, II, 112, 2

201. Ovide, Pontiques, III, 5, 7.
202. Ibid., II, 3, 5.

203. Z. Popova, 1968, p. 60. C'est la seule épitaphe versifiée du $\mathrm{I}^{\mathrm{er}} \mathrm{s}$. qui peut être datée facilement.

204. Forschungen in Ephesos, III, p. 112, 22.

205. Sardis, VII, 1, 36.

206. SEG, XLIII, 775

207. $A E, 1912,135$

208. Sénèque, De la colère, II, 5, 5.

209. P. Hermann, 2007, p. 23-24.

210. Nous ne connaissons pas la nature de cette construction. 
avancer la date de 46/47, s'il s'agit de Claude, ou de 67, s'il s'agit de Néron. Les lacunes sur le nombre d'itérations de la puissance tribunicienne et sur le nombre de consulats ne permettent pas d'être plus précis. Néanmoins, il est préférable de retenir la date de 46/47, car en 67 , Cotta aurait été trop âgé, même si un de ses ancêtres du $\mathrm{I}^{\mathrm{er}} \mathrm{S}$. av. J.-C., Messalla Rufus, vécut très longtemps ${ }^{211}$. L'absence de la mention d'un quelconque titre ou rappel de sa fonction de proconsul d'Asie laisse penser qu'il a agi à titre privé, afin de satisfaire son ami et a gardé des contacts en Asie mineure, plus de dix années après son proconsulat. L'inscription montre les liens existants entre Cotta et le pouvoir impérial. Peut-être était-il encore proche du pouvoir à l'époque de Claude? Signalons qu'à ce moment, le princeps est marié à Messalline, issue de la gens des Valerii. Enfin, nous savons dorénavant que Cotta était encore vivant au moins jusqu'en 47 , alors que la dernière attestation littéraire, dans l'œuvre tacitéenne, date de 32 et que son proconsulat se place vraisemblablement en 35/36.

Parmi les critères de distinction de l'élite dirigeante romaine, la clientèle et le patronat sont particulièrement prégnants et Cotta a pu bénéficier des réseaux déjà tissés par sa gens. Toutefois, cette emprise sociale n'existerait pas sans une fortune et un patrimoine conséquents, qui participent à la définition de l'identité nobiliaire.

\section{LA RICHESSE ET LE PATRIMOINE DE COTTA}

Cotta a probablement dû hériter du patrimoine foncier et immobilier de son père adoptif, mais le patrimoine des Aurelii Cottae est peu connu. On ne peut guère tirer profit d'une source littéraire dont l'action se déroule artificiellement en 7776 av. J.-C. : le traité du De natura deorum de Cicéron est réputé se dérouler dans l'exèdre de la domus de C. Aurelius Cotta, le consul de $75^{212}$. La localisation de cette demeure n'est pas certaine, mais elle doit être proche de l'aedes honoris et uirtutis, près de la Porte Capène ${ }^{213}$. Toutefois, rien

211. Macrobe, Saturnales, I, 9, 14. Il a été augure pendant cinquante-cinq ans.

212. Cicéron, De la nature des dieux, I, 15.

213. D. Palombi, 1995, p. 65.

214. La forme Athalis Ilua est également acceptée par l'édition Teubner de J. A. Richmond (1990, p. 37).

215. Ovide, Pontiques, II, 3, 83-84. n'indique une transmission de cette propriété à Cotta Messallinus.

Un passage d'une lettre d'exil d'Ovide est sujet à polémique: Vltima me tecum uidit maestisque cadentes / Excepit lacrimas Athalis Ilua ${ }^{214}$ genis $^{215}$. Cotta disposerait d'un patrimoine foncier à Ilva, soit sur l'île d'Elbe ${ }^{216}$, dernier lieu de rencontre entre lui et le poète, en 8 , avant le départ en exil ${ }^{217}$. Dans cette optique, il est légitime de penser que cette entrevue eut lieu dans une propriété du sénateur. Or, cette localisation est fondée sur la restitution de Ethalis Ilua. J. Carcopino ${ }^{218}$ a montré que les manuscrits les plus récents offraient la forme Ithalis ora, ensuite transformée en Italica ora, restitution rejetée par Carcopino, tandis que la plus ancienne tradition présente la forme d' Fithalis Ilua, faisant penser à Fthalis Ilua, soit l'île d'Elbe aethalienne. Toutefois, cette identification doit être rejetée car la rencontre n'aurait pas pu avoir lieu dans la mer Tyrrhénienne, puisque Ovide devait partir via l'Adriatique. J. Carcopino propose la forme d'Aletha Silua, soit les bois d'Aletha-Alesium. Aletha se trouve à quelques dizaines de kilomètres d'Hydrus, à l'extrême sud-est de l'Italie, port permettant le plus court trajet vers l'autre rive. L'hypothèse d'une propriété de Cotta sur l'île d'Elbe ne peut pas être retenue.

Le patrimoine de Cotta n'est guère identifiable. Il n'a certainement pas hérité des jardins de Lucullus sur le Pincio, appartenant à son père naturel Messalla Corvinus ${ }^{219}$. Si le patrimoine foncier et la fortune sont bien des marqueurs identitaires de la noblesse, les témoignages épigraphiques ou littéraires ne permettent pas d'en identifier les composantes avec précision.

\section{COTTA ET LA DIMENSION CULTURELLE DES VALERII MESSALLAE}

Parmi les marqueurs culturels de la légitimité nobiliaire, l'éloquence tient une place primordiale. L'empreinte paternelle est tenace et il est difficile pour $\operatorname{Cotta}^{220}$ de se départir du souvenir de l'élo-

216. A. M. Andermahr, 1998, p. 75

217. E. Groag, PIR ${ }^{2}, 1933, \mathrm{n}^{\circ} 1488$.

218. J. Carcopino, 1962, p. 174.

219. Sur ce point, voir notamment H. Broise et V. Jolivet, 1987, p. 747-761 et 1996, p. 86-87.

220. Sur les mentions de l'éloquence de Cotta, $c f$. A. Balbo, 2007, $\mathrm{n}^{\circ} 23$. 
quence de son père, Messalla Corvinus, reconnu comme un des meilleurs orateurs de son temps ${ }^{221}$. D'ailleurs, Ovide se plaît à mettre en relation les deux personnages ${ }^{222}$ et Cotta ne serait pas indigne de l'éloquence paternelle. Cette qualité ferait partie de son héritage familial, en quelque sorte ${ }^{223}$. Même si l'éloquence n'était plus un moyen de gouvernement, elle n'en demeurait pas moins importante, notamment lors des discours prononcés au Sénat ou sur le forum, comme l'atteste une lettre d'exil d'Ovide ${ }^{224}$. Néanmoins, ce sont les talents oratoires du frère de Cotta, Messallinus, qui sont surtout mis en exergue par le poète ${ }^{225}$.

A contrario, Tacite utilise le thème de l'éloquence de Cotta pour montrer la décadence de l'éloquence antique : Cotta a hérité de ce talent de Messalla Corvinus, mais a dépouillé l'art oratoire de son rôle républicain et de sa dignité, en le tournant dorénavant vers l'adulatio. Il n'hésite pas à rappeler que certains orateurs ont gardé leur honneur, comme M. Lepidus ${ }^{226}$. L'auteur a dû écrire en réaction à la tradition indulgente présentant notamment Cotta comme un mécène, un protecteur des lettres ${ }^{227}$. Cette image négative se retrouve dans les scholies de Perse. L'occurrence concernant Messalla ${ }^{228}$, présente dans l'œuvre de Perse, est conforme à ce jugement. Cornutus ${ }^{229}$ propose donc une explication. D'abord, la descendance aux yeux malades de Messalla désigne Cotta. Celui-ci aurait été touché par une maladie oculaire et par un problème aux paupières, peutêtre par un strabisme divergent. Le Commentum
Cornuti insiste surtout sur la dépravation du fils cadet de Corvinus. Cette connotation péjorative atteste la vision d'un scholiaste chrétien sur une réalité romaine devenue incompréhensible.

Cotta a été un homme de lettres, utilisé comme source par Pline l'Ancien pour le livre XIV et pour le suivant dont le thème est la connaissance des arbres fruitiers. Dans le livre X, l'auteur le cite dans son exposé sur les oiseaux, plus particulièrement sur la manière de les cuisiner. En effet, le patricien aurait eu l'idée d'accommoder des pattes d'oie avec des crêtes de $\operatorname{coq}^{230}$. Ses autres travaux littéraires ne sont guère connus, malgré une allusion d'Ovide ${ }^{231}$.

Un autre aspect de la dimension culturelle doit être apprécié, celui du mécénat et l'existence d'un cercle littéraire. D'abord, Cotta ne se contentait pas d'écrire, puisqu'il était aussi amateur de poésie ${ }^{232}$. D'ailleurs, Ovide consacre une épître à la présentation d'un Art poétique chez les Gètes à $\operatorname{Cotta}^{233}$, le jugeant digne et capable de juger l'œuvre à sa juste valeur. $\mathrm{H}$. Bardon cite deux passages de Juvénal ${ }^{234}$, où le second mécène mentionné serait Aurelius Cotta ${ }^{235}$, mais nous avons déjà vu qu'il faut plutôt l'identifier avec son fils.

Enfin, un dernier aspect mêlant littérature, mécénat et amitié vaut d'être précisé : les liens entre Cotta et Ovide. D'après une lettre d'exil du poète, ils sont anciens: Quod eras aliis factus, mihi natus amicus/ Quodque tibi in cunis oscula prima dedi,/ Quod cum uestra domus teneris mihi semper ab annis/
221. Les sources sont nombreuses à en faire l'écho, notamment Horace, Art poétique, 366-373 de son vivant. Le souvenir de son éloquence perdure après sa mort, voir notamment : Quintilien, Institution oratoire, X, 1, 113. Quintilien établit une correspondance entre la nobilitas et l'éloquence de Messalla Corvinus : at Messala nitidus et candidus et quodam modo praeferens in dicendo nobilitatem suam, uiribus minor.

222. Ovide, Pontiques, III, 5, 7.

223. Sur l'importance de l'éloquence et sur l'hérédité de cette uirtus, consulter Chr. Badel, 2005, p. 173-175.

224. Ovide, Pontiques, III, 5, 3-16. Le discours aurait été tenu devant la iudicio centumuirali (vers 23-24). Le poète lisait le discours à voix haute.

225. Ovide, Tristes, IV, 4, 1-8 notamment.

226. Tacite, Annales, IV, 20, 2.

227. Juvénal, Satires, VII, 94-95. Cf. R. Syme, 1986, p. 239.

228. Perse, Satires, II, 71-72 : Quin damus id superis, de magna quod dare lancel Non possit magni Messalae lippa propago? "Que ne donnons-nous aux dieux ce que ne pourrait leur donner sur un grand plat la progéniture aux yeux malades du grand
Messalla?» (Traduction A. Cartault, C.U.F.). Ce passage est également cité dans les Institutiones grammaticae (II, 9, 472) de Priscianus.

229. Commentum Cornuti in Persium in Satiram II, 71-72 (voir note 105).

230. Pline l'Ancien, Histoire naturelle, X, 27, 52

231. Ovide, Pontiques, III, 5, 35-36 : Redde uicem, nec rara tui monimenta laboris/ Accipiant nostrae grata futura manus. "Paie-moi de retour et que mes mains reçoivent souvent les témoignages de ton travail, qui me seront toujours agréables» (traduction J. André, C.U.F.). Nous ne connaissons que ces ouvrages spécifiques. Nous ne savons pas s'il a écrit de la poésie ou des traités, comme son père. H. Bardon pense qu'il s'agit d'épigrammes, mais sans véritable explication. H. Bardon, 1956, p. 52.

232. Ovide, Tristes, IV, 5, 11-12.

233. Pour de plus amples informations sur ce point, $c f$. D. Voisin, 2000, p. 416-417.

234. Juvénal, Satires, V, 109-110 et VII, 94-95.

235. H. Bardon, 1956, p. 52. 
Culta sit, esse uetus me tibi cogit onus ${ }^{236}$. Le poète précise qu'il a toujours préféré Cotta ${ }^{237}$. Ovide a noué très jeune des liens avec Messalla Corvinus, même s'il ne fait pas vraiment partie du cercle du patricien. Ce sont les informations présentes dans les Tristes $^{238}$ et dans les Pontiques ${ }^{239}$ qui apportent le plus de renseignements. Le poète rappelle que Messalla fut le premier à croire à son talent ${ }^{240}$. R. Syme s'est interrogé sur cette mention si tardive de Messalla. En effet, pourquoi avoir attendu la mort de Corvinus et son exil pour parler de ce soutien précoce? Aucune œuvre de jeunesse ne s'en fait l'écho. Pourtant, ce ne serait pas étonnant, puisque les œuvres de jeunesse ne mentionnent aucun patron ${ }^{241}$, comme c'est le cas dans les Amours. Un autre point émerge des poèmes : en ajoutant la mention qu'il connut Cotta à sa naissance vers 14 av. J.-C., le poète indique qu'il faisait déjà partie de l'entourage du patricien ${ }^{242}$. Enfin, il ne faut pas oublier qu'Ovide aspire à bénéficier de la clémence impériale. C'est notamment pourquoi il écrit à Cotta, dont les liens étroits avec l'empereur sont connus. En rappelant l'ancienneté des relations entre Ovide et le père de Cotta, le poète espère que Cotta agisse pour favoriser le retour d'exil.

Les relations entre Cotta et Ovide dépassent très largement le cadre du mécénat et de la sociabilité littéraire, puisque c'est un des derniers à entretenir une correspondance avec l'exilé. Sur les trente lettres des trois premiers livres des Pontiques, six sont adressées à Cotta ${ }^{243}$. C'est ainsi le principal destinataire des lettres de l'exilé et il est qualifié de sodalis ${ }^{244}$. D'accoutumée, ce terme désigne des êtres ayant sensiblement le même âge, les mêmes intérêts et des fortunes équivalentes ${ }^{245}$. Or, ils ont presque trente ans d'écart et Cotta est un membre de l'aristocratie sénatoriale. Ensuite, la lettre V, 9 est consacrée à l'amitié existant entre les deux personnages. Ovide n'hésite pourtant pas à adresser des reproches, à peine voilés, à l'encontre du sénateur. Ainsi, dans la lettre des Pontiques I, 5, Ovide parle des relations amicales entre les deux personnages au passé ${ }^{246}$ et il laisse paraître sa déception, puisque Cotta avait même proposé de lui apporter une aide matérielle dans le cas où sa fortune serait confisquée ${ }^{247}$. En dépit d'une amitié ancienne, Cotta ne pouvait pas se compromettre politiquement en demandant la clémence impériale pour le poète, même sous le principat de Tibère.

En tant que descendant d'une lignée patricienne séculaire, le fils cadet de Messalla Corvinus était porteur d'une légitimité spécifique, celle qui avait fait la puissance de sa famille sous la République. Mais l'instauration du Principat a changé la donne politique. Né sous le règne d'Auguste, Cotta a fait la majorité de sa carrière politique sous le principat de Tibère, dont il était un ami de longue date et faisait partie de l'entourage proche du Prince. L'analyse des identités nobiliaire et patricienne a permis de montrer que les marqueurs étaient globalement les mêmes : les revendications généalogiques, les sacerdoces, le patrimoine, la clientèle... Il n'y a pas eu de rupture radicale. La principale mutation est décelable dans le domaine politique où nous assistons à la naissance d'une nobilitas impériale, dont Cotta est un des meilleurs représentants. Pour asseoir le régime du Principat, Auguste s'est appuyé sur le patriciat. La restauration de ce dernier a permis d'assurer la continuité des marqueurs de l'identité patricienne. Toutefois, l'adhésion au nouveau régime et les nouveaux comportements politiques sont passés pour de l'adulatio pure et simple et le tableau négatif dressé
236. Ovide, Pontiques, II, 3, 71-74: «Tu es devenu un ami, pour moi tu le fus à ta naissance, que je t'ai donné dans ton berceau mes premiers baisers, et que, pour avoir toujours honoré ta famille depuis mes tendres années, je suis par force un fardeau pour toi depuis longtemps" (traduction J. André, C.U.F.).

237. Ovide, Pontiques, II, 3, 81-82.

238. C'est son premier recueil de lettres d'exil (ce n'est pas un exil proprement dit car il conserve ses biens, il est préférable de parler de relegatio). Elles sont composées en trois ans et demi, entre le début du mois de décembre 8 et 12 ap. J.-C.

239. Contrairement au premier recueil, les noms des destinataires sont énoncés. Les épîtres sont rédigées entre 13 et 16 .
240. Ovide, Pontiques, I, 7, 27-28 et II, 3, 75-78.

241. R. Syme, 1978, p. 75-76. R. Syme invite à minimiser les relations entre le mécène et le poète. Effectivement, elles ne sont pas exclusives car Ovide a participé à différents cercles littéraires : R. Syme, 1978, p. 117.

242. R. Syme, 1978, p. 76.

243. Ovide, Pontiques, I, 5; I, 9; II, 3; II, 8; III, 2 et III, 5.

244. Dans la lettre IV, 5 des Tristes, les deux personnages sont qualifiés de sodales (vers 1).

245. D. Voisin, 2000, p. 415.

246. Ovide, Pontiques I, $5,1$.

247. Ovide, Tristes, IV, 5, 7-8. 
par Tacite en est l'expression la plus visible. L'historiographie s'est donc détournée de ce noble, alors que son cas montre l'adaptation de l'antique noblesse aux profondes mutations opérées. Cotta illustre parfaitement les fondements de la nobilitas romaine et son moyen de gouverner, décrits par R. Syme : "Les nobiles maniaient et détenaient trois armes, la famille, l'argent et l'alliance politique $»^{248}$.

Cyrielle LANDREA

\section{Bibliographie}

A. M. Andermahr, Totus in Praediis. Senatorischer Grundbesitz in Italien in der frühen und hohen Kaiserzeit, Bonn, 1998.

J. Arce et J. González (dir.), Estudios sobre la Tabula Siarensis, Madrid, 1988.

Chr. Badel, La noblesse de l'Empire romain. Les masques et la vertu, Paris, 2005.

A. Balbo, I frammenti degli oratori romani dell'età augustea e tiberiana, parte seconda, Alessandria, 2007.

H. Bardon, La littérature latine inconnue. Tome II, l'époque impériale, Paris, 1956.

R. Baudry, Patriciens et nobles à Rome, d'une identité à l'autre?, dans Hypothèses, 2006, p. 155-167.

H. Broise et V. Jolivet, Recherches sur les jardins de Lucullus, dans $L^{\prime}$ Vrbs. Espace urbain et histoire ${ }^{\text {er }}$ s. av. - III s. ap. J.-C., Paris, 1987, p. 747-761.

H. Broise et V. Jolivet, Horti : Valerius Messalla Corvinus, dans LTVR, III, Rome, 1996, p. 86-87.

T. R. S. Broughton, The magistrates of the Roman Republic, I, New York, 1951

W. H. Buckler et D. M. Robinson, Sardis, VII, Greek and Latin inscriptions, I, Leyde, 1932.

J. L. Butrica, Messalla and the Principate, dans C. Deroux (dir.), Studies in Latin literature and Roman history, VII, Bruxelles, p. 279-296.

A. Caballos, W. Eck et F. Fernandez, El senadoconsulto de Gneo Pisón padre, Séville, 1996.

L. Canina, La prima parte della Via Appia dalla Porta Capena a Boville, Rome, 1853.

J. Carcopino, L'exil d'Ovide, dans Rencontres de l'histoire et de la littérature romaines, Paris, 1953, p. 115-129.

J. Carcopino, Un prétendu séjour d'Ovide à l'île d'Elbe, dans Comptes rendus de l'Académie des inscriptions et belles-lettres, 1962, 106, p. 173-174.

M. Cébeillac-Gervasoni, Les quaestores principis et candidati aux $I^{e r}$ et $I I^{e}$ s. de l'Empire, Milan, 1973.

V. Chapot, Données nouvelles sur la prosopographie de l'Asie proconsulaire, dans Mélanges en hommage à la mémoire de Fr. Martroye, Paris, 1940, p. 81-92.

M. H. Crawford (dir.), Roman statutes, I, Londres, 1996.

J. Crook, Consilium Principis. Imperial Councils and Counsellors from Augustus to Diocletian, Cambridge, 1955.

A. Degrassi, Osservazioni su alcuni consoli suffetti dell'età di Augusto e Tiberio, dans Epigraphica, 8, 1946, p. 34-39.

S. J. De Laet, Notes sur la carrière de deux sénateurs romains - M. Aurelius Cotta Maximus Messalinus, dans L'Antiquité Classique, 6, 1937, p. 138-140.

S. Demougin, L'ordre équestre sous les Julio-Claudiens, Paris, 1988 (Collection de l'École française de Rome, 108).

S. Demougin, Prosopographie des chevaliers romains julioclaudiens (43 av. J.-C. - 70 ap. J.-C.), Paris, 1992 (Collection de l'École française de Rome, 153).

W. Eck, A. Caballos et F. Fernández, Das senatus consultum de Cn. Pisone patre, Munich, 1996.

L. Fanizza, Il senato e la prevenzione del crimen repetundarum in età tiberiana, dans Labeo, 23, 2, 1977, p. 199-214.

J. Ferguson, A prosopography to the poems of Juvenal, Bruxelles, 1987

F. Fernández et J. González, Tabula Siarensis, dans Iura, 32, 1981, p. 1-36.

M.-C. Ferriès, Les partisans d'Antoine (des orphelins de César aux complices de Cléopâtre), Bordeaux, 2007. 
A. Fraschetti (dir.), La commemorazione di Germanico nella documentazione epigrafica. Tabula Hebana e Tabula Siarensis, Rome, 2000.

E. Galletier, Étude sur la poésie funéraire romaine d'après les inscriptions, Paris, 1922.

J. González, Tabula Siarensis, Fortunales Siarenses et municipia ciuium romanorum, dans Zeitschrift für Papyrologie und Epigraphik, 55, 1984, p. 55-100.

P. Graindor, Athènes de Tibère à Trajan, Le Caire, 1931.

L. Grifi, Parte prima. Dal consolato di L. Munazio Planco e C. Silio fino a quello di M. Valerio e M. Aurelio, dans Dissertazioni della pontifica accademia romana di archeologia, 1855, p. 491-497.

E. Groag, M. Aurelius Cotta, PIR², I, A, no 1487, 1933.

E. Groag, M. Aurelius Cotta Maximus Messalinus, PIR ${ }^{2}$, I, A, $\mathrm{n}^{\circ} 1488,1933$.

E. Groag, Q. Caecilius Metellus Creticus Silanus, RE, III, A, 1, s.v. Caecilius nº 90, 1897, col. 1212.

R. Hanslik, M. Valerius Messalla Corvinus, RE, VIII, A, 1, s.v. Valerius no 261, 1955, col. 131-157.

P. Hermann, New documents from Lydia, Vienne, 2007.

M. Hofmann, Ti. Plautius Silvanus Aelianus, RE, XXI, I, s.v. Plautius $n^{\circ} 47,1951$, col. 35-40.

Fr. Hurlet, Les sénateurs dans l'entourage d'Auguste et de Tibère. Un complément à plusieurs synthèses récentes sur la cour impériale, dans Revue de philologie, de littérature et d'histoire anciennes, 74, 2000, p. 123-150.

Fr. Hurlet, Le centre du pouvoir : Rome et la cour impériale aux deux premiers siècles de notre ère, dans N. Belayche (dir.), Rome, les Césars et la Ville aux deux premiers siècles de notre ère, Rennes, 2001, p. 159-183.

Fr. Hurlet, L'aristocratie augustéenne et la Res publica restituta, dans Fr. Hurlet, et B. Minéo (dir.), Le principat d'Auguste. Réalités et représentations du pouvoir. Autour de la Res publica restituta, Rennes, 2009, p. 73-99.

M. Kajava, Roman senatorial women and the Great East. Epigraphic evidence from the Republican and Augustan period, dans H. Solin et M. Kajava (dir.), Roman Eastern policy and other studies in Roman history, Helsinki, 1990, p. 59-124.

M. Kajava, Vesta and Athens, dans O. Salomies (dir.), The Greek East in the Roman context, Helsinki, 2001, p. 71-94.

E. Klebs, M. Aurelius Cotta Maximus Messalinus, PIR, saec. I, II, III, A, 1897, n 1236.

P. Le Roux, Siarum et la Tabula Siarensis : statut politique et honneurs religieux en Bétique sous Tibère, dans J. Arce et J. González (dir.), Estudios sobre la Tabula Siarensis, Madrid, 1988, p. 21-33.

C. Letta, Un nuovo frammento dei Fasti di Salii Palatini, dans G. Paci (dir.), Miscellanea epigrafica in onore di Lidio Gasperini, tome 1, Rome, 2000, p. 521-539.

B. Levick, Tiberius the politician, Londres, 1976.

M. Macciocca, Cottae sepulcrum, in LTVR, Suburbium, II, Rome, 2004, p. 166.

A. J. Marshall, Roman Women and the Provinces, dans Ancient History, 6, 1975, p. 109-128.

F. Millar, Imperial ideology in the Tabula Siarensis, dans J. Arce et J. González (dir.), Estudios sobre la Tabula Siarensis, Madrid, 1988, p. 11-19.

F. Münzer, De gente Valeria, Berlin, 1891.

F. Münzer, $M^{\prime}$. Valerius Maximus Messalla, RE, VIII, A, I, s.v. Valerius no 247, 1955, col. 123-125.

J. H. Oliver, Aurelius Cotta, dans The American Journal of Philology, 92 (1), 1971, p. 55.

D. Palombi, Domus : C. Aurelius Cotta, dans Lexicon topographicum urbis Romae, II, Rome, 1995, p. 65.

Z. Popova, Pour dater les carmina latina epigraphica Buecheler 990, 55 et 960, dans Eirene, Studia Graeca et Latina, 7, 1968, p. 57-66.

M.-H. Prévost, Les adoptions politiques à Rome sous la République et le Principat, Paris, 1949.

M.-Th. Raepsaet-Charlier, Prosopographie des femmes de l'ordre sénatorial (I $I^{e r}-I^{e}$ s.), Louvain, 1987.

Y. Rivière, Les délateurs sous l'Empire romain, Paris, 2002 (Bibliothèques des Écoles françaises d'Athènes et de Rome, 311).

R. S. Rogers, Der Prozess des Cotta Messalinus, dans Hermes, 68, 1933, p. 121-123.

L. Ross Taylor, Trebula Suffenas and the Plautii Silvani, Memoirs of the American Academy in Rome, 24, 1956, p. 7-30.

J. Rüpke, Fasti sacerdotum. Die Mitglieder der Priesterschaften und das sakrale Funktionspersonal römischer, griechischer, orientalischer und jüdisch-christlicher Kulte in der Stadt Rom von 300 v. Chr. bis 499 n. Chr., Teil 2 : Biographien, Stuttgart, 2005.

J. Rüpke, Römische Priester in der Antike. Ein biographisches Lexikon, Stuttgart, 2007.

O. Salomies, Adoptive and Polyonymous Nomenclature in the Roman Empire, Helsinki, 1992 (Commentationes Humanarum Litterarum, 97).

O. Salomies, Choosing a cognomen in Rome. Some Aspects, dans H. M. Schellenberg, V. E. Hirschmann et A. Krieckhaus (dir.), Essays in Honour of Anthony R. Birley on his seventieth Birthday, Gdansk, 2008, p. 79-91.

J. C. Saquete, Las vírgenes vestales. Un sacerdocio femenino en la religión pública romana, Madrid, 2000.

J. Scheid, Les Frères arvales : recrutement et origine sociale sous les empereurs julio-claudiens, Paris, 1975. 
M. Aurelius Cotta Maximus Messallinus : un noble sous les Julio-Claudiens

C. M. Stibbe, G. Colonna, C. De Simone et H. C. Versnel, Lapis Satricanus. Archaeological, epigraphical, linguistic and historical aspects of the new inscription from Satricum, La Haye, 1980.

R. Syme, Personal Names in Annales I-VI, dans Journal of Roman Studies, 39, 1949, p. 6-18.

R. Syme, Some Pisones in Tacitus, dans Journal of Roman Studies, 46, 1956, p. 17-21.

R. Syme, The Roman Revolution, Oxford, 1939 (cité dans la traduction française, La révolution romaine, Paris, 1967).

R. Syme, History in Ovid, Oxford, 1978.

R. Syme, The Augustan aristocracy, Oxford, 1986.

P. Tansey, Messalla Corvinus and the Bellum Siculum, dans Latomus, 66, 2007, p. 882-890.

A. Valvo, M. Valerio Messalla Corvino negli studi più recenti, dans $A N R W$, II, 30, 3, W. Haase (dir.), Berlin-New York, 1983, p. 1663-1681.

U. Vogel-Weidemann, Miscellanea zu den Proconsules von Africa und Asia zwischen 14 und 68 n. Chr., dans Zeitschrift für Papyrologie und Epigraphik, 46, 1982, p. 271-294.

U. Vogel-Weidemann, Die Statthalter von Africa und Asia in den Jahren 14-68 n. Chr. : eine Untersuchung zum Verhältnis Princeps und Senat, Bonn, 1982.
D. Voisin, Les cercles littéraires à Rome à l'époque d'Auguste, 2000 (thèse de l'université de Lille 3).

H. Volkmann, $M^{\prime}$. Valerius Maximus, RE, VIII, A, I, s.v. Valerius $\mathrm{n}^{\circ} 243$, 1955, col. 116-120.

H. Volkmann, P. Valerius Poplicola, RE, VIII, A, I, s.v. Valerius $\mathrm{n}^{\circ}$ 302, 1955, col. 180-189.

H. Volkmann, M. Valerius Corvus, RE, VII, A, III, s.v. Valerius $\mathrm{n}^{\circ} 137,1948$, col. 2413-2418.

D. von Lunzer, M. Valerius Messalla Messallinus, RE, VIII, A, 1, s.v. Valerius no 264, 1955, col. 159-162.

P. von Rohden, Aurelius Cotta, RE, IV, s.v. Aurelius no 93, 1896, col. 2481.

P. von Rohden, L. Aurelius Cotta, RE, IV, s.v. Aurelius $\mathrm{n}^{\circ}$ 102, 1896, col. 2485-2487.

P. von Rohden, M. (Aurelius) Cotta, RE, IV, s.v. Aurelius $\mathrm{n}^{\circ} 108,1896$, col. 2489.

P. von Rohden, M. (Aurelius) Cotta, RE, IV, s.v. Aurelius $\mathrm{n}^{\circ} 109,1896$, col. 2489.

P. von Rohden, M. Aurelius Cotta, RE, IV, s.v. Aurelius $\mathrm{n}^{\circ}$ 110, 1896, col. 2489-2490.

P. von Rohden, M. Aurelius Cotta Maximus Messallinus, RE, IV, s.v. Aurelius no 111, 1896, col. 2490-2491.

A. Winterling, Aula Caesaris. Studien zur Institutionalisierung des römischen Kaiserhofes in der Zeit von Augustus bis Commodus (31 v. Chr. - 192 n. Chr.), Munich, 1999. 


\section{Annexe}

TABLEAU GÉNÉALOGIQUE SIMPLIFIÉ DES VALERII MESSALLAE AU I ${ }^{\text {er }}$ S. AV. ET AU Ir S. AP. J.-C.

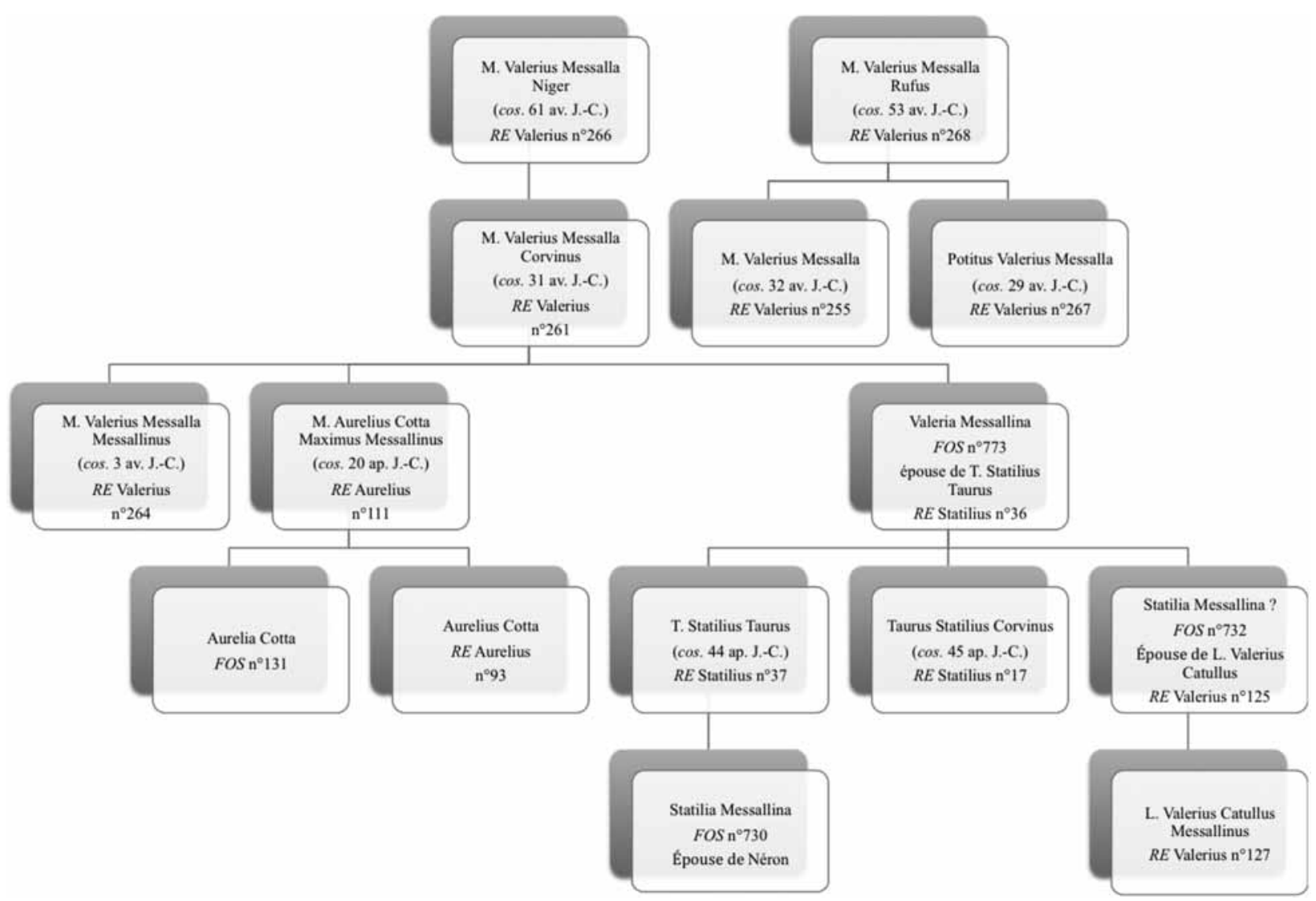


